\title{
ON THE ACCUMULATION OF SEPARATRICES BY INVARIANT CIRCLES
}

\author{
A. KATOK* AND R. KRIKORIAN
}

\begin{abstract}
Let $f$ be a smooth symplectic diffeomorphism of $\mathbb{R}^{2}$ admitting a (non-split) separatrix associated to a hyperbolic fixed point. We prove that if $f$ is a perturbation of the time- 1 map of a symplectic autonomous vector field, this separatrix is accumulated by a positive measure set of invariant circles. On the other hand, we provide examples of smooth symplectic diffeomorphisms with a Lyapunov unstable non-split separatrix that are not accumulated by invariant circles.
\end{abstract}

\section{INTRODUCTION}

A theorem by M.R. Herman, "Herman's last geometric theorem", cf. [11], [12]), asserts that if a smooth orientation and area preserving diffeomorphism $f$ of the 2-plane $\mathbb{R}^{2}$ (or the 2-cylinder $\mathbb{R} / \mathbb{Z} \times \mathbb{R}$ ) admits a KAM circle $\Sigma$ (by definition, a smooth invariant curve 1 on which the dynamics of $f$ is conjugated to a Diophantine translation) then this KAM circle is accumulated by other KAM circles the union of which has positive 2-dimensional Lebesgue measure in any neighborhood of $\Sigma$. In this paper we investigate whether such a phenomenon holds if, instead of being a KAM circle, the invariant set $\Sigma$ is a separatrix of a hyperbolic fixed (or periodic) point of $f$.

More precisely, we consider the following situation. Let $f: \mathbb{R}^{2} \rightarrow \mathbb{R}^{2}$, $f:(x, y) \mapsto f(x, y), f(0,0)=(0,0)$ be a smooth diffeomorphism which is symplectic w.r.t. the usual symplectic form $\omega=d x \wedge d y\left(f^{*} \omega=\omega\right)$. We assume that $o:=(0,0)$ is a hyperbolic fixed point of $f$ (the matrix $D f(o) \in S L(2, \mathbb{R})$ has distinct real eigenvalues) and that there exists an $f$-invariant compact connected set $\Sigma \ni o$ such that $\Sigma \backslash\{o\}$ is a nonempty connected 1-dimensional manifold included in both the stable and unstable manifolds $W_{f}^{s}(o), W_{f}^{u}(o)$ associated to $o$ :

$$
\forall(x, y) \in \Sigma, \quad \lim _{n \rightarrow \pm \infty} f^{n}(x, y)=o .
$$

\footnotetext{
* A preliminary version of this paper was discussed by the authors some months before Anatole Katok passed away in April 2018.

A. Katok was partially supported by NSF grant DMS 1602409. R. Krikorian was supported by a Chaire d'Excellence LABEX MME-DII, the project ANR BEKAM : ANR15-CE40-0001 and an AAP project from CY Cergy Paris Université.

${ }^{1}$ Isotopic in $\mathbb{R}^{2} \backslash\{o\}$ to a circle centered at the origin in the case $f: \mathbb{R}^{2} \rightarrow \mathbb{R}^{2}$ or isotopic to $\mathbb{R} / \mathbb{Z} \times\{0\}$ in the cylinder case.
} 


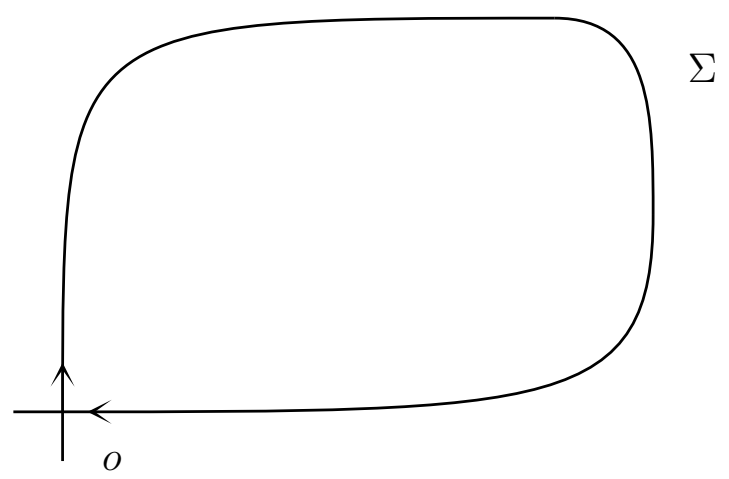

FiguRE 1. A (non-split) separatrix

Note that since $o$ is $f$-hyperbolic, $\Sigma$ is homeomorphic to a circle and $\Sigma \backslash\{o\}$ coincides with one of the two connected components of $W_{f}^{s}(o) \backslash\{o\}$ (resp. $\left.W_{f}^{u}(o) \backslash\{o\}\right)$. We shall say that $\Sigma$ is a separatrix of $f$ associated to the hyperbolic fixed point $o$ or, without referring to the hyperbolic fixed point $o$, that $\Sigma$ is a separatrix of $f$.

Examples of such diffeomorphisms $f$ can be obtained in the following way. Let $X_{0}$ be a smooth autonomous Hamiltonian vector field of the form

$$
X_{0}=J \nabla H_{0}, \quad J=\left(\begin{array}{cc}
0 & -1 \\
1 & 0
\end{array}\right)
$$

where $H_{0}: \mathbb{R}^{2} \rightarrow \mathbb{R}$, of the form

$$
H_{0}(x, y)=Q_{0}(x, y)+O^{3}(x, y), \quad \lambda \in \mathbb{R}^{*},
$$

(we can assume without loss of generality $\lambda>0$ ) is a smooth function. The time-1 map $f_{0}:=\phi_{X_{0}}^{1}$ of $X_{0}$ is a Hamiltonian (in particular symplectic) diffeomorphism of $\mathbb{R}^{2}$ admitting $o$ as a hyperbolic fixed point. We assume that it has a separatrix $\Sigma \ni o$ of the form

$\Sigma \backslash\{o\}=\left\{\phi_{X_{0}}^{t}(p), t \in \mathbb{R}\right\}, \quad$ for some $p \in \mathbb{R}^{2} \backslash\{o\}$ s.t. $\quad \lim _{t \rightarrow \pm \infty} \phi_{X_{0}}^{t}(p)=o$.

We now consider a smooth time-dependent Hamiltonian vector field $Y$ : $\mathbb{R} / \mathbb{Z} \times \mathbb{R}^{2} \rightarrow \mathbb{R},(t,(x, y)) \mapsto Y(t, x, y)$ which is 1 -periodic in $t$, symplectic w.r.t. $(x, y)$ and tangent to $\Sigma \backslash\{o\}$ :

$$
\forall t \in \mathbb{R} / \mathbb{Z}, \forall(x, y) \in \Sigma, \quad \operatorname{det}\left(X_{0}(x, y), Y(t, x, y)\right)=0 .
$$

One can for example choose $Y(t, x, y)=J \nabla F(t, x, y)$ where $F: \mathbb{R} / \mathbb{Z} \times \mathbb{R}^{2} \rightarrow$ $\mathbb{R}$ is a smooth time-dependent Hamiltonian that satisfies

$$
\forall t \in \mathbb{R} / \mathbb{Z}, \forall(x, y) \in \Sigma, \quad F(t, x, y)=F(t, 0,0) .
$$


Note that since $o$ is a hyperbolic fixed point of $X_{0}$ one has for all $t, Y(t, o)=$ 0 . For $\varepsilon \in \mathbb{R}$ define the 1-periodic in $t$ symplectic vector field $\mathbb{R}^{2} \rightarrow \mathbb{R}^{2}$

$$
X_{\varepsilon}^{t}(x, y):=X_{\varepsilon}(t, x, y)=X_{0}(x, y)+\varepsilon Y(t, x, y) .
$$

For $\varepsilon$ small enough, the time-0-to- 1 map 2

$$
f_{\varepsilon}=\phi_{X_{\varepsilon}}^{1,0}
$$

of the symplectic vector field $X_{\varepsilon}$ is a symplectic diffeomorphism of $\mathbb{R}^{2}$ admitting $O$ as a hyperbolic fixed point and still $\Sigma$ as a separatrix. Note that $f_{\varepsilon}$ is a Hamiltonian diffeomorphism (for more details on Hamiltonian diffeomorphisms see [16]).

Here is the analogue of the aforementioned Last Geometric Theorem of Herman:

Theorem A. For any $r \in \mathbb{N}^{*}$ there exists $\varepsilon_{r}>0$, such that, for any $\varepsilon \in$ ]$-\varepsilon_{r}, \varepsilon_{r}$ [, there exists a set of $f_{\varepsilon}$-invariant $C^{r}$ KAM-circles accumulating the separatix $\Sigma$ and which covers a set of positive Lebesgue measure of $\mathbb{R}^{2}$ in any neighborhood of $\Sigma$.

Let us clarify some points in the preceding statement.

By a $C^{r}$ circle, $r \geqslant 0$, we mean a $C^{r}$ non-self-intersecting closed curve (or equivalently, if $r \geqslant 1$, a nonempty compact connected 1-dimensional $C^{r}$ submanifold of $\mathbb{R}^{2}$ ) which is isotopic in $\mathbb{R}^{2} \backslash\{o\}$ to the separatrix $\Sigma$. Such a set $\Gamma$ is invariant by $f_{\varepsilon}$ if $f_{\varepsilon}(\Gamma)=\Gamma$.

We say that a set $\mathcal{G}$ of $f_{\varepsilon}$-invariant circles accumulates the set $\Sigma$ if for any $\xi>0$, the set of $\Gamma \in \mathcal{G}$ such that $\operatorname{dist}(\Gamma, \Sigma)<\xi$ is not empty, where dist denotes the Hausdorff distance

$$
\operatorname{dist}(A, B)=\max \left(\sup _{a \in A} d(a, B), \sup _{b \in B} d(b, A)\right)
$$

(here $d(x, C)=\inf _{c \in C}\|x-c\|_{\mathbb{R}^{2}}$ ).

The $f_{\varepsilon}$-invariant circles obtained in Theorem $\mathrm{A}$ are KAM circles: the restrictions of $f_{\varepsilon}$ on each of these curves are $C^{r}$ circle diffeomorphisms that are conjugated to Diophantine 3 translations. In our case, the exponent of the Diophantine condition can be choosen to be independent of $\varepsilon$ (it depends only on $\lambda$ ).

Remark 1.1. On the other hand, and this is a difference with the situation of Herman's Last Geometric Theorem, the constants of these Diophantine

\footnotetext{
${ }^{2}$ If $X(t, z)$ is a time dependent vector field the time-s-to- $t$ map of $X$ is defined by $\phi_{X}^{t, s}(z(s))=z(t)$ for any $z(\cdot)$ solution of $\dot{z}(t)=X(z(t))$. When $X$ is time-independent the notation $\phi_{X}^{t}$ stands for $\phi_{X}^{t, 0}$.

${ }^{3} \mathrm{~A}$ real number $\alpha$ is Diophantine if there exist positive constants $\kappa, \tau$ such that for any $(p, q) \in \mathbb{Z} \times \mathbb{N}^{*},|\alpha-(p / q)| \geqslant \kappa / q^{\tau}$. The constants $\tau$ and $\kappa$ are respectively called the exponent and the constant of the Diophantine condition. The set of Diophantine numbers with fixed exponent $\tau>2$ has full Lebesgue measure if the constant is not specified and positive measure if the constant is also fixed (and small).
} 
numbers are arbitrarily small. Moreover, as these circles accumulate the separatrix, their $C^{2}$-norm must explode.

Remark 1.2 . The phase space $\mathbb{R}^{2}$ can be replaced by the cylinder $\mathbb{R} / \mathbb{Z} \times \mathbb{R}$ in the statement of the Main Theorem.

The smallness condition in Theorem $\mathrm{A}$ is indeed necessary as shows the following theorem.

Let $\Delta_{\Sigma}$ be the bounded connected component of $\mathbb{R}^{2} \backslash \Sigma$.

Theorem B. There exists a smooth symplectic diffeomorphism $f: \mathbb{R}^{2} \rightarrow \mathbb{R}^{2}$ admitting a separatrix $\Sigma$ which is included in an open set $W$ of $\Sigma \cup \Delta_{\Sigma}$ that contains no f-invariant circle in $W \backslash \Sigma$.

The situation described in the above Theorems $\mathrm{A}, \mathrm{B}$ is not generic. Indeed, as Poincaré discovered, in general, the stable and unstable manifolds of a hyperbolic fixed or periodic point of a symplectic map intersect transversally (one usually refers to this phenomenon as the spiltting of separatri$c e s$ ), a fact that forces the dynamics of $f$ to be "quite intricate". This was Poincaré's key argument in his proof of the fact that the Three-body problem in Celestial Mechanics does not admit a complete set of independent commuting first integrals. Later, Smale [18] showed that this splitting of separatrices has an even more striking consequence on the dynamics of $f$, namely the existence of a horseshoe, that is, a uniformly hyperbolic $f$ invariant compact set (locally maximal) with positive topological entropy 4 and on which the dynamics of $f$ is "chaotic" (isomorphic to a 2-sided shift). A consequence of the splitting of a separatrix is thus the existence of a Birkhoff instability zone (open region without invariant circles) in the vicinity of this split separatrix (see [10] for a detailed exposition on the topic). In some sense, Theorem $\mathrm{A}$ shows that in the perturbative situation (1.2)-(1.3) ( $\varepsilon$ small enough) the splitting of separatrices is essentially the only mechanism responsible for the creation of instability zones. On the other hand, in a "non perturbative" situation, Theorem B points in the opposite direction. Figures 4, 7 illustrate the role that plays the smallness assumption in Theorem $\mathrm{A}$ (or its absence in Theorem $\mathrm{B}$ ).

On the proofs of Theorems $A$, B. - As suggests Remark 1.1, the invariant circles of Theorem A cannot be obtained directly via a classical KAM approach. On the other hand, the existence of the (non split) separatrix $\Sigma$ allows to associate to each diffeomorphism $f_{\varepsilon}$ a regular diffeomorphism $f_{\varepsilon}^{\circ}$, defined on a standard open annulus and preserving a finite probability measure, to which one can apply Moser's or Rüssmann's Invariant (or translated) curve Theorem [15], [17] (see Section 6). The thus obtained invariant curves for $\stackrel{\circ}{\varepsilon}_{\varepsilon}$ yield invariant curves for $f_{\varepsilon}$. The construction of the diffeomorphism

\footnotetext{
${ }^{4} \mathrm{By}$ a result of the first author [13, in this situation, positive topological entropy is indeed equivalent to the existence of a horseshoe.
} 
$\AA_{\varepsilon}$ is done as follows. We first make preliminary reductions involving some Birkhoff and symplectic Sternberg-like Normal Forms (Section 2) to have a control on the dynamics in some neighborhood of the hyperbolic fixed point $o$ (Section 3). This allows us to define in Section 4 a first return map $\hat{f}_{\varepsilon}$ for $f_{\varepsilon}$, in a fundamental domain $\mathcal{F}_{\varepsilon}$ the boundaries of which can be glued together to obtain an open abstract cylinder (or annulus). This abstract cylinder can be uniformized to become a standard annulus and the first return map $\hat{f}_{\varepsilon}$ then becomes a regular diffeomorphism $\bar{f}_{\varepsilon}$ of a standard annulus (preserving some probability measure). This is done in Section 5. We call normalization (see Section 5.3) the uniformization operation and we say that $\bar{f}_{\varepsilon}$ is the renormalization 5 of $f_{\varepsilon}$. The dynamics of $\bar{f}_{\varepsilon}$ is closely related to that of $f_{\varepsilon}$ in the sense that the existence of invariant curves for $\bar{f}_{\varepsilon}$ translates into a similar statement for $f_{\varepsilon}$ (see Section 7 ). The renormalized diffeomorphism $\bar{f}_{\varepsilon}$ has a large twist (this is a reminiscence of the hyperbolicity of $f_{\varepsilon}$ at $o$ ) and we are thus led to rescale it to obtain the aforementioned diffeomorphism $\stackrel{\circ}{f}_{\varepsilon}$ which is now a small $C^{r}$-perturbation of an integrable twist map (this is where the smallness assumption of Theorem $\mathrm{A}$ appears) with controled twist (see Section 6). The proof of Theorem A is completed in Section 8 ,

To prove Theorem B ( $c f$. Section 9) we construct a symplectic diffeomorphism $f$ (named $f_{\text {pert }}$ in that section) so that the associated renormalized diffeomorphism $\bar{f}$ has an orbit accumulating the boundary of the aforementioned annulus: this prevents the existence of $\bar{f}$-invariant curves close to this boundary and therefore of $f$-invariant curves close to the separatrix $\Sigma$.

The technique6 we use to prove Theorem A might be useful to study the dynamics of symplectic twist maps with zero topological entropy (to which extent are they integrable?7) and the construction of Theorem $\mathrm{B}$ might give a hint to provide examples of smooth twist maps admitting isolated invariant circles with irrationa rotation number (if they exist, these curves bound two instability zones).

Acknowledgments. The second author (RK) wishes to thank Bassam Fayad and the referee for their thorough reading of a preliminary version of this paper and their very useful comments.

\footnotetext{
${ }^{5}$ The term renormalization in this paper has the same acceptation as in the theories of circle diffeomorphisms, holomorphic germs or quasi-periodic cocycles; cf. [24, 23], 14, [6.

${ }^{6}$ We note that the authors of [21 introduce the "separatrix map" constructed by a glueing construction to investigate the size of the instability zones. Our approach here, which is focused on a renormalization point of view, is different.

${ }^{7}$ Angenent, 11, proves they are $C^{0}$-integrable in the sense that for any rotation number one can find a $C^{0}$-invariant curve with this rotation number. Can one prove $C^{k}$ integrability? The word "integrable" is meant in a broad sense

${ }^{8}$ A modification of the example of Theorem B yields examples of such isolated invariant curves with rational rotation numbers. For the existence of curves with irrational rotation number in low regularity and related results see [2], 3], 4], [5].
} 


\section{NORMAL Forms}

The main result of this Section is the following Sternberg-like symplectic Normal Form theorem (Proposition 2.1) that will allow us in Section 3 to control the long-time dynamics of $f_{\varepsilon}$ in a neighborhood of the hyperbolic point $o$. This will be useful when we shall define first return maps for $f_{\varepsilon}$ in convenient fundamental domain, see Section 4 .

Let $f_{\varepsilon}$ defined by (1.3), (1.2) .

Proposition 2.1. For any $k \in \mathbb{N}^{*}$ large enough, there exists $\varepsilon_{k}>0$ for which the following holds. There exist a smooth family $\left(q_{\varepsilon, k}\right)_{\varepsilon \in I}$ ( $I \ni 0$ some open interval of $\mathbb{R}$ ) of polynomials $q_{\varepsilon, k}(s)=\lambda s+O\left(s^{2}\right) \in \mathbb{R}[s]$ and a continuous family $\left(\Theta_{\varepsilon, k}\right)_{\varepsilon \in I}$ of symplectic $C^{k}$-diffeomorphism of $\mathbb{R}^{2}$ such that $\Theta_{\varepsilon, k}(o)=o$, $D \Theta_{\varepsilon, k}(o)=i d$ and on a neighborhood $V_{k}$ of o one has, provided $\left.\varepsilon \in\right]-\varepsilon_{k}, \varepsilon_{k}[$ :

$$
\text { on } \begin{aligned}
V_{k}, \quad f_{\varepsilon, k} \underset{\text { defin. }}{=} \Theta_{\varepsilon, k} \circ f_{\varepsilon} \circ \Theta_{\varepsilon, k}^{-1} \\
=\phi_{J \nabla Q_{\varepsilon, k}}^{1}, \quad \text { where } \quad Q_{\varepsilon, k}(x, y)=q_{\varepsilon, k}(x y)
\end{aligned}
$$

and

$$
\text { on } V_{k}, \quad\left(\Theta_{0, k}\right)_{*} X_{0}=J \nabla Q_{0, k}
$$

Note that $o$ is still a hyperbolic fixed point of $f_{\varepsilon, k}$ and that

$$
\Sigma_{\varepsilon, k}:=\Theta_{\varepsilon, k}(\Sigma)
$$

is still a separatrix for $f_{\varepsilon, k}$.

Reduction of Theorem A to Theorem 2.1. - After applying Proposition 2.1 we are thus left with a family $\left(f_{\varepsilon, k}\right)$ of $C^{k}$ - symplectic diffeomorphisms, each $f_{\varepsilon, k}$ being conjugated to $f_{\varepsilon}$ and admitting a separatrix $\Sigma_{\varepsilon, k}$. Since the conclusions of Theorem $\mathrm{A}$ are clearly invariant by conjugation, to prove Theorem $\mathrm{A}$ we just need to prove that if $k \geqslant r$ and $\varepsilon$ is small enough, each separatrix $\Sigma_{\varepsilon, k}$ is accumulated by a set of positive measure of KAM-circles for $f_{\varepsilon, k}$. This is the content of Theorem 2.1 below, that we shall apply to the family of $C^{k}$-diffeomorphisms $f_{\varepsilon, k}$ defined by (2.4), (1.3), (1.2), but that holds for any family (that we still denote in what follows $\left(f_{\varepsilon}\right)_{\varepsilon \in I}$ to alleviate the notations) of symplectic $C^{k}$-diffeomorphisms satistying the following hypothesis.

Let $\left(f_{\varepsilon}\right)_{\varepsilon \in I},(I \ni 0$ open interval of $\mathbb{R})$ be a family of $C^{k}$-symplectic diffeomorphisms of $\mathbb{R}^{2}$ that satisfies:

(H1) Each $f_{\varepsilon}$ has a (non split) separatrix $\Sigma_{\varepsilon}$ associated to the hyperbolic point $o$.

(H2) The map $I \ni \varepsilon \rightarrow f_{\varepsilon}-i d \in C^{k}\left(\mathbb{R}^{2}, \mathbb{R}^{2}\right)$ is continuous (the norm on $C^{k}$ is the usual $C^{k}$-norm); 
(H3) On some neighborhood $V$ of $o$, each $f_{\varepsilon}$ coincides with the time-1 map of a symplectic vector field $J \nabla Q_{\varepsilon}(x, y)$ where $Q_{\varepsilon}(x, y)=q_{\varepsilon}(x y)$, $q_{\varepsilon} \in C^{k+1}\left(\mathbb{R}^{2}\right)$

$$
q_{\varepsilon}(t)=\lambda t+O^{2}(t), \quad \lambda>0 .
$$

(H4) On $\mathbb{R}^{2}, f_{0}=\phi_{X_{0}}^{1}$ where $X_{0}=J \nabla H_{0}$ is a hamiltonian vector field that coincides with $J \nabla Q_{0}$ on $V$.

Remark 2.1. On $V$ the orbits of $f_{\varepsilon \mid V}=\phi_{J \nabla Q_{\varepsilon}}^{1}$ are pieces of hyperbolae $\{x y=\mathrm{cst}\}$ (condition $(H 3)$. .

When $\varepsilon=0$, for any $z \in\{x y=c\} \cap V, N \in \mathbb{Z}$ such that $f_{0}^{N}(z) \in V$ one has $f_{0}^{N}(z) \in\{x y=c\} \cap V$ (condition (H4)).

Remark 2.2. The intersection $\Sigma_{\varepsilon} \cap V$ is the union

$$
\Sigma_{\varepsilon} \cap V=\left(W_{f_{\varepsilon}}^{s}(o) \cap V\right) \cup\left(W_{f_{\varepsilon}}^{u}(o) \cap V\right)
$$

and

$$
W_{f_{\varepsilon}}^{s}(o) \cap V=(\mathbb{R} \times\{0\}) \cap V, \quad W_{f_{\varepsilon}}^{u}(o) \cap V=(\{0\} \times \mathbb{R}) \cap V .
$$

One then has:

Theorem 2.1. There exists $k_{0} \in \mathbb{N}$ for which the following holds. Let $k \geqslant k_{0}+2$ and let $\left(f_{\varepsilon}\right)_{\varepsilon \in I}$ be a family of $C^{k}$-symplectic diffeomorphisms of $\mathbb{R}^{2}$ satisfying the previous conditions (H1), (H2), (H3), (H4). Then, there exists $\varepsilon_{1}>0$ such that for any $\left.\varepsilon \in\right]-\varepsilon_{1}, \varepsilon_{1}$ [ the diffeomorphism $f_{\varepsilon}$ admits a set of positive Lebesgue measure of invariant $C^{k-k_{0}-2}$-circles in any neighborhood of the separatrix $\Sigma_{\varepsilon}$.

Moreover, if $k-k_{0}-2 \geqslant k_{1}$ ( $k_{1}$ depending only on $\lambda$ ) these circles are KAM-circles.

We shall give the proof of Theorem 2.1 in Section 8 ,

The proof of Proposition 2.1 occupies the rest of this Section. It will be based on a first reduction obtained by performing some steps of Birkhoff Normal Forms (Proposition 2.3) and then the application of various Sternberg like Normal Forms (Corollary 2.4 and Proposition 2.5).

2.1. Birkhoff Normal Form for the time-periodic vector field $X_{\varepsilon}^{t}$. A preliminary step in Sternberg's classical Linearization Theorem is to first conjugate the considered system (diffeomorphism or vector field) defined in the neighborhood of the hyperbolic fixed point $o$ to a system which is tangent to an integrable model to some high enough order. This is what we do in this subsection and in a symplectic framework (see Proposition 2.3) by using Birkhoff Normal Form techniques. 
2.1.1. Periodically forced vector fields. Let $X: \mathbb{R} \times \mathbb{R}^{2} \ni(t, x) \mapsto X^{t}(z):=$ $X(t, z) \in \mathbb{R}^{2}$ be a smooth time dependent symplectic vector field: for each $t$ the 1 -form $i_{X_{t}} \omega$ is closed (and hence locally exact). For $t, s \in \mathbb{R}$ we denote by $\phi_{X}^{t, s}$ the flow of $X$ between times $s$ and $t$ when it is defined (see footnote 2 for the definition of $\phi_{X}^{t, s}$ ). If $t \mapsto g^{t}(\cdot)$ is a one-parameter family of symplectic diffeomorphisms one has

$$
g^{t} \circ \phi_{X}^{t, s} \circ\left(g^{s}\right)^{-1}=\phi_{\tilde{X}}^{t, s}
$$

where $\tilde{X}:(t, z) \mapsto \tilde{X}^{t}(z):=\tilde{X}(t, z)$ is the smooth time dependent symplectic vector field

$$
\tilde{X}^{t}=\partial_{t} g^{t} \circ\left(g^{t}\right)^{-1}+\left(g^{t}\right)_{*} X^{t}
$$

Conversely, if (2.8) is satisfied then so is (2.7). Note that if $g^{t}$ depends 1-periodically on $t$ then (2.7) yields the more classical conjugation equation

$$
g \circ \phi_{X}^{1,0} \circ g^{-1}=\phi_{\tilde{X}}^{1,0}
$$

where $g=g^{0}=g^{1}\left(g^{t}\right.$ is 1-periodic in $\left.t\right)$.

Assume now that $X^{t}$ depends 1-periodically in $t$ and in a smooth way on a small parameter $\varepsilon \in \mathbb{R}$; we furthermore assume that it is of the form

$$
X_{\varepsilon}^{t}(z)=J \nabla H_{\varepsilon}^{t}(z)
$$

where $\left(z=\left(z_{1}, z_{2}\right) \in \mathbb{R}^{2}\right)$

$$
H_{\varepsilon}^{t}(z)=\lambda_{\varepsilon}(t) z_{1} z_{2}+O^{3}(z), \quad \int_{\mathbb{T}} \lambda_{\varepsilon}(t) d t>0, \quad \lambda_{0}(t)=\lambda \in \mathbb{R}_{+}^{*},
$$

$H_{\varepsilon}: \mathbb{R} / \mathbb{Z} \times \mathbb{R}^{2} \rightarrow \mathbb{R}, H_{\varepsilon}:(t, z) \mapsto H_{\varepsilon}(t, z):=H_{\varepsilon}^{t}(z)$ being a smooth function. Assume also that for some $j \in \mathbb{N}^{*}$

$$
g_{\varepsilon}^{t}(z)=\phi_{J \nabla G_{\varepsilon}^{t}}^{1}(z)=i d+O^{j}(z), \quad G_{\varepsilon}^{t}(z)=O^{j+1}(z)
$$

where $G: I \times \mathbb{R} / \mathbb{Z} \times\left(\mathbb{R}^{2}, o\right) \ni(\varepsilon, t, z) \mapsto G_{\varepsilon}(t, z):=G_{\varepsilon}^{t}(z) \in \mathbb{R}$ is a smooth function. Then, one has

$$
\begin{gathered}
\partial_{t} g_{\varepsilon}^{t} \circ\left(g_{\varepsilon}^{t}\right)^{-1}=J \nabla \partial_{t} G_{\varepsilon}^{t}+O^{j+1}(z) \\
\left(g_{\varepsilon}^{t}\right)_{*} X_{\varepsilon}^{t}=J \nabla H_{\varepsilon}^{t} \circ\left(g_{\varepsilon}^{t}\right)^{-1}=J \nabla H_{\varepsilon}^{t}+J \nabla\left\{G_{\varepsilon}^{t}, H_{\varepsilon}^{t}\right\}+O^{j+1}(z)
\end{gathered}
$$

(here $\{A, B\}$ denotes the Poisson bracket $\{A, B\}=\langle\nabla A, J \nabla B\rangle$ ) so that $\tilde{X}_{\varepsilon}^{t}$ defined by (2.8) is of the form

$$
\tilde{X}_{\varepsilon}^{t}=J \nabla \tilde{H}_{\varepsilon}^{t}
$$

with

$$
\begin{aligned}
\tilde{H}_{\varepsilon}^{t} & =H_{\varepsilon}^{t}+\partial_{t} G_{\varepsilon}^{t}+\left\{G_{\varepsilon}^{t}, H_{\varepsilon}^{t}\right\}+O^{j+2}(z) \\
& =H_{\varepsilon}^{t}+\partial_{t} G_{\varepsilon}^{t}+\left\{G_{\varepsilon}^{t}, H_{2, \varepsilon}^{t}\right\}+O^{j+2}(z)
\end{aligned}
$$

where we have denoted $H_{2, \varepsilon}^{t}\left(z_{1}, z_{2}\right)=\lambda_{\varepsilon}(t) z_{1} z_{2}$. 
If in the preceding equation one chooses $G_{\varepsilon}^{t}=G_{\varepsilon, 2}^{t}$ with $G_{\varepsilon, 2}^{t}(z)=a_{\varepsilon, 0}(t) z_{1} z_{2}$ where $a_{\varepsilon, 0}$ is the 1-periodic function defined by

$$
a_{\varepsilon, 0}(t)=-\int_{0}^{t}\left(\lambda_{\varepsilon}(s)-\int_{\mathbb{T}} \lambda_{\varepsilon}(u) d u\right) d s
$$

one has

$$
\tilde{H}_{\varepsilon}^{t}(z)=\bar{\lambda}_{\varepsilon} z_{1} z_{2}+O^{3}(z)
$$

where $\bar{\lambda}_{\varepsilon}=\int_{\mathbb{T}} \lambda_{\varepsilon}(t) d t$. In other words, performing a change of coordinates (2.8) on $X_{\varepsilon}^{t}$ with $g_{\varepsilon}^{t}=g_{\varepsilon, 2}^{t}=\phi_{J \nabla G_{\varepsilon, 2}^{t}}^{1}$ we can assume that in (2.10) $\lambda_{\varepsilon}(t)$ does not depend on $t$

$$
H_{\varepsilon}^{t}(z)=\lambda_{\varepsilon} z_{1} z_{2}+O^{3}(z), \quad \lambda_{\varepsilon} \in \mathbb{R}_{+}^{*}
$$

(we write $\lambda_{\varepsilon}$ in place of $\bar{\lambda}_{\varepsilon}$ ).

2.1.2. Birkhoff Normal Form. Having put $H_{\varepsilon}^{t}$ under the form (2.14), we now eliminate by successive conjugations (2.8) non-diagonal higher order terms in $z$ from $H_{\varepsilon}^{t}$ (note that they depend on $t$ ).

The following lemma describes this elimination procedure.

Lemma 2.2. Let $j \in \mathbb{N}, j \geqslant 2$. Assume that for some polynomials $q_{\varepsilon}(s)=$ $\lambda s+O\left(s^{2}\right) \in \mathbb{R}[s]$ of degree $\leqslant[j / 2]$ depending smoothly on $\varepsilon$

$$
H_{\varepsilon}^{t}(z)=q_{\varepsilon}\left(z_{1} z_{2}\right)+O^{j+1}(z) .
$$

Then, there exist a smooth family $\left(\tilde{q}_{\varepsilon}\right)_{\varepsilon}$ of polynomials $\tilde{q}_{\varepsilon}(s)=\lambda s+O\left(s^{2}\right) \in$ $\mathbb{R}[s]$ of degree $\leqslant[(j+1) / 2]$ and a smooth family of smooth maps $G_{\varepsilon}$ : $\mathbb{R} / \mathbb{Z} \times\left(\mathbb{R}^{2}, o\right) \ni(t, z) \rightarrow G_{\varepsilon}(t, z)=G_{\varepsilon}^{t}(z) \in \mathbb{R}^{2}$ such that on a neighborhood of $o$

$$
\left\{\begin{array}{l}
G_{\varepsilon}^{t}(z)=O^{j+1}(z) \\
H_{\varepsilon}^{t}(z)+\partial_{t} G_{\varepsilon}^{t}(z)+\left\{G_{\varepsilon}^{t}, H_{\varepsilon}^{t}\right\}(z)=\tilde{q}_{\varepsilon}\left(z_{1} z_{2}\right)+O^{j+2}(z) .
\end{array}\right.
$$

Moreover, if for $\varepsilon=0, H_{0}^{t}$ does not depend on $t$, one can choose $G_{0}^{t}$ to be independent of $t$.

Proof. See the Appendix, Section A.

Let now $X_{\varepsilon}^{t}$ be the family of vector fields of (1.2).

Proposition 2.3. For any $N \geqslant 1$ there exist an open neighborhood $V_{N}$ of o,

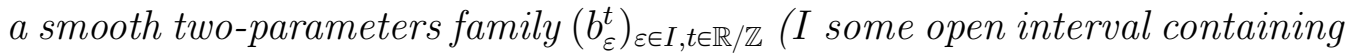
$0)$ of smooth symplectic diffeomorphisms $b_{\varepsilon}^{t}:\left(\mathbb{R}^{2}, o\right) \bigcirc$ satisfying $b_{\varepsilon}^{t}(o)=o$, $D b_{\varepsilon}^{t}(o)=i d$ and a smooth family of polynomials $q_{\varepsilon, N}(s)=\lambda s+O\left(s^{2}\right)$ of degree $\leqslant[(N+1) / 2]$, such that for any $\varepsilon \in I, t \in \mathbb{R} / \mathbb{Z},(x, y) \in V_{N}$ one has

$$
\begin{aligned}
X_{\varepsilon}^{(1), t} \underset{\text { defin. }}{=}\left(\partial_{t} b_{\varepsilon}^{t}\right) \circ\left(b_{\varepsilon}^{t}\right)^{-1}+\left(b_{\varepsilon}^{t}\right)_{*} X_{\varepsilon}^{t} \\
\quad=J \nabla Q_{\varepsilon, N}+O^{N+1}(x, y) \quad \text { with } \quad Q_{\varepsilon, N}(x, y)=q_{\varepsilon, N}(x y)
\end{aligned}
$$

and for $\varepsilon=0, b_{0}^{t}$ is independent of $t$. 
Proof. Applying the preceding Lemma 2.2 and relations (2.8)-(2.12) inductively (starting from (2.14) ) we thus construct polynomials $q_{\varepsilon, j}$ of degree $\leqslant[j / 2](j \geqslant 2)$ and functions $G_{\varepsilon, j}^{t}=O^{j+1}(z)$ such that if one defines

$$
b_{\varepsilon}^{t}=g_{\varepsilon, N}^{t} \circ \ldots \circ g_{\varepsilon, 2}^{t}=i d+O^{2}(z), \quad g_{\varepsilon, j}^{t}=\phi_{J \nabla G_{\varepsilon, j}^{t}}^{1}=i d+O^{j}(z)
$$

one has

$$
\begin{aligned}
\tilde{X}_{\varepsilon}^{t}: & =\partial_{t} b_{\varepsilon}^{t} \circ\left(b_{\varepsilon}^{t}\right)^{-1}+\left(b_{\varepsilon}^{t}\right)_{*} X_{\varepsilon}^{t} \\
& =J \nabla Q_{\varepsilon, N}+O^{N+1}(z), \quad \text { with } \quad Q_{\varepsilon, N}(z)=q_{\varepsilon, N}\left(z_{1} z_{2}\right)
\end{aligned}
$$

all dependences on $\varepsilon$ being smooth. Moreover, if $X_{0}^{t}$ is independent of $t$ the diffeomorphism $b_{0}^{t}$ is independent of $t$.

Remark 2.3. Note that since $b_{0}^{t} \equiv b_{0}$ is independent of $t$, the vector field

$$
X_{0}^{(1)}=\left(b_{0}\right)_{*} X_{0}
$$

is autonomous.

\subsection{Symplectic Sternberg theorem for the autonomous vector field}

$X_{0}^{(1)}$. We shall need a symplectic version of the famous theorem by S. Sternberg (on smooth linearization of hyperbolic germs of smooth vector fields, see [19]) as proved in [7] or [8] (see also [20]). We follow here the exposition of [7].

Let $Z_{i}, i=1,2$ be two symplectic smooth autonomous vector fields such that for some $\lambda \in \mathbb{R}^{*}$ and $N \in \mathbb{N}$ one has

$$
\left\{\begin{array}{l}
Z_{i}(x, y)=-\lambda x \frac{\partial}{\partial x}+\lambda y \frac{\partial}{\partial y}+O^{2}(x, y)(i=1,2) \\
Z_{1}(x, y)-Z_{2}(x, y)=O^{N+1}(x, y) .
\end{array} .\right.
$$

Theorem 2.2 ([7], Theorem 1.2). There exist positive constants $A, B$ for which the following holds. Let $m \in \mathbb{N}^{*}$ large enough and $N=[(m+B) / A]+$ $1 \geqslant 1$. If (2.16) is satisfied then there exists a $C^{m}$ symplectic change of coordinates $S_{0}:\left(\mathbb{R}^{2}, 0\right) \bigcirc$ such that on a neighborhood of o

$$
\left\{\begin{array}{l}
\left(S_{0}\right)_{*} Z_{1}=Z_{2} \\
S_{0}(o)=0, \quad D S_{0}(o)=i d
\end{array}\right.
$$

We apply the preceding theorem to the case $Z_{1}=X_{0}^{(1)}$ and $Z_{2}=J \nabla Q_{0, N}$ $\left(X_{0}^{(1)}, Q_{0, N}\right.$ given by Proposition 2.3 when $\left.\varepsilon=0\right)$. In view of Proposition 2.3, the condition (2.16) is satisfied and we hence get a symplectic diffeomorphism $S_{0}$ satisfying $S_{0}(o)=0, D S_{0}(o)=i d$ and such that on a neighborhood of $O$

$$
\left(S_{0}\right)_{*} X_{0}^{(1)}=J \nabla Q_{0, N}
$$

For each value of $t \in \mathbb{R} / \mathbb{Z}$ and $\varepsilon \in I$, the diffeomorphism $\left(S_{0} \circ b_{\varepsilon}^{t}\right)$ fixes the origin and its derivative at the origin is the identity. It can thus be extended as a symplectic $C^{m}$-diffeomorphism $R_{\varepsilon}^{t}$ of $\mathbb{R}^{2}$ ( $c f$. Lemma B.1). Note that the dependence of $R_{\varepsilon}^{t}$ w.r.t. $t$ is smooth and 1-periodic $(t \in \mathbb{R} / \mathbb{Z})$. We now define 
on $\mathbb{R} / \mathbb{Z} \times \mathbb{R}^{2}$ the time-periodic vector field $X_{\varepsilon}^{(2)}:(t,(x, y)) \in(\mathbb{R} / \mathbb{Z}) \times \mathbb{R}^{2} \rightarrow \mathbb{R}^{2}$ by

$$
X_{\varepsilon}^{(2), t} \underset{\text { defin. }}{=}\left(\partial_{t} R_{\varepsilon}^{t}\right) \circ\left(R_{\varepsilon}^{t}\right)^{-1}+\left(R_{\varepsilon}^{t}\right)_{*} X_{\varepsilon}^{t}
$$

and we observe that on a neighborhood of $O$

$$
\left(R_{0}^{t}\right)_{*} X_{0}=X_{0}^{(2)}=J \nabla Q_{0, N}
$$

Since the conjugacy relation (2.18) is equivalent to (see subsection 2.1.1)

$$
\forall t, s, \quad R_{\varepsilon}^{t} \circ \phi_{X_{\varepsilon}}^{t, s} \circ\left(R_{\varepsilon}^{s}\right)^{-1}=\phi_{X_{\varepsilon}^{(2)}}^{t, s}
$$

we get by taking $t=1, s=0$ and setting $R_{\varepsilon}:=R_{\varepsilon}^{1}=R_{\varepsilon}^{0}$, the following corollary:

Corollary 2.4. If $m \in \mathbb{N}^{*}$ is large enough and $N=[(m+B) / A]+1$, there exists a smooth family $\left(R_{\varepsilon}\right)$ of $C^{m}$ symplectic diffeomorphisms of $\mathbb{R}^{2}$ such that $R_{\varepsilon}(o)=o, D R_{\varepsilon}(o)=i d$ and on a neighborhood of $o$

$$
\begin{aligned}
f_{\varepsilon}^{(1)}: & \underset{\text { defin. }}{=} R_{\varepsilon} \circ f_{\varepsilon} \circ\left(R_{\varepsilon}\right)^{-1} \\
& =\phi_{J \nabla Q_{\varepsilon, N}}^{1}+O^{N+1}(x, y) .
\end{aligned}
$$

Moreover

$$
\left(R_{0}\right)_{*} X_{0}=J \nabla Q_{0, N}
$$

Note that the last equation shows that

$$
f_{0}^{(1)}=\phi_{J \nabla Q_{0, N}}^{1}
$$

\subsection{Symplectic Sternberg Normal Form for the diffeomorphism} $f_{\varepsilon}^{(1)}$. Theorem 2.2 has a version for smooth germs of symplectic diffeomorphisms which are hyperbolic at the origin, this is Theorem 1.1 of [7]. In our paper we shall need a parametric version of that result, which is not explicitly stated in [7] but that can be checked after close examination of the proof.

Proposition 2.5. There exist constants $A_{1}, B_{1}$ depending on $\lambda \in \mathbb{R}^{*}$ such that the following holds. Let $m \in \mathbb{N}^{*}$ large enough and $N=[m / 2]-3$. If $\left(g_{1, \varepsilon}\right)_{\varepsilon \in I}$ and $\left(g_{2, \varepsilon}\right)_{\varepsilon \in I}$ ( $I \ni 0$ some open interval of $\mathbb{R}$ ) are two continuous (w.r.t. $\varepsilon \in I)$ families of $C^{m}$ symplectic diffeomorphisms $\left(\mathbb{R}^{2}, o\right) \bigcirc$ such that

$$
\left\{\begin{array}{l}
\forall \varepsilon, \quad g_{1, \varepsilon}(o)=g_{2, \varepsilon}(o)=o \\
D g_{1,0}(o)=\operatorname{diag}\left(\lambda, \lambda^{-1}\right) \quad(\text { is hyperbolic }) \\
g_{1, \varepsilon}(x, y)=g_{2, \varepsilon}(x, y)+O^{N+1}(x, y) \\
g_{1,0}=g_{2,0}
\end{array}\right.
$$


then, there exists a continuous family $\left(S_{\varepsilon}^{(1)}\right)_{\varepsilon}$ (w.r.t. $\varepsilon \in I$ small enough), of $C^{k}$ symplectic diffeomorphisms such that $S_{\varepsilon}^{(1)}(o)=o, D S_{\varepsilon}^{(1)}(o)=i d$ with $k=\left[N A_{1}-B_{1}\right]-1$ and

$$
\left\{\begin{array}{l}
S_{\varepsilon}^{(1)} \circ g_{1, \varepsilon} \circ\left(S_{\varepsilon}^{(1)}\right)^{-1}=g_{2, \varepsilon} \\
S_{0}^{(1)}=i d .
\end{array}\right.
$$

2.4. Proof of Proposition 2.1. It will be a consequence of Corollary 2.4 and Proposition 2.5,

We first choose $N$ so that $k=\left[N A_{1}-B_{1}\right]-1$ and we define $m$ by $N=[(m+B) / A]+1$. If $k$ is large enough $m$ will satisfy the assumption of Corollary 2.4. We then apply Proposition 2.5 to $g_{1, \varepsilon}=f_{\varepsilon}^{(1)}, g_{2, \varepsilon}=\phi_{J \nabla Q_{\varepsilon, N}}^{1}$ which satisfy (2.23) (note that (2.20) is satisfied). This provides us with a continuous family $\left(S_{\varepsilon}^{(1)}\right)_{\varepsilon}$ of $C^{k}$ symplectic diffeomorphisms defined in a fixed neighborhood of $o$ such that $S_{\varepsilon}^{(1)}(o)=o, D S_{\varepsilon}^{(1)}(o)=i d$ and on a neighborhood of $o$

$$
\left\{\begin{array}{l}
S_{\varepsilon}^{(1)} \circ f_{\varepsilon}^{(1)} \circ\left(S_{\varepsilon}^{(1)}\right)^{-1}=\phi_{J \nabla Q_{\varepsilon, N}}^{1} \\
S_{0}^{(1)}=i d .
\end{array}\right.
$$

We can extend these $S_{\varepsilon}^{(1)}$ as symplectic $C^{k}$ diffeomorphisms $S_{\varepsilon}^{(2)}$ of $\mathbb{R}^{2}$ which depend continuously on $\varepsilon$ (cf. Lemma B.1). We then define

$$
\Theta_{\varepsilon, k}=S_{\varepsilon}^{(2)} \circ R_{\varepsilon}
$$

and we observe that on a neighborhood of $o$

$$
\left\{\begin{array}{l}
\Theta_{\varepsilon, k} \circ f_{\varepsilon} \circ \Theta_{\varepsilon, k}^{-1}=\phi_{J \nabla Q_{\varepsilon, N}}^{1} \\
\left(\Theta_{\mathbf{0}, k}\right)_{*} X_{0}=J \nabla Q_{0, N}
\end{array}\right.
$$

indeed, the first equality comes from (2.19) and the first equation of (2.24), while the second is a consequence of (2.21) and the second equation of (2.24).

To conclude the proof we rename $q_{\varepsilon, N}, Q_{\varepsilon, N}$ as $q_{\varepsilon, k}, Q_{\varepsilon, k}$.

Note: From now on, and till the end of Section 8 , we shall work in the setting of Theorem 2.1 with a family of $C^{k}$ symplectic diffeomorphisms satisfying conditions $(H 1),(H 2),(H 3),(H 4)$.

\section{Dynamics in a neighborhood of the ORIGiN}

The purpose of this Section is to estimate the time spent by the orbits of the flow $\Phi_{J \nabla Q_{\varepsilon}}^{t}$ in the neighborhood $V$ of the hyperbolic point $o$.

To do that, we perform one more change of coordinates. 
Let us define the following diffeomorphisms $\Xi_{1}, \Xi_{2}$

$$
\begin{cases}\forall(x, y) \in \mathbb{R}_{+}^{*} \times \mathbb{R}, & \Xi_{1}(x, y)=(\ln x, x y), \\ \forall(x, y) \in \mathbb{R} \times \mathbb{R}_{+}^{*}, & \Xi_{2}(x, y)=(-\ln y, x y) .\end{cases}
$$

Since $d(\ln x) \wedge d(x y)=d(-\ln y) \wedge d(x y)=d x \wedge d y$ we see that $\Xi_{i}, i=1,2$, are symplectic.

Let $I_{1}, I_{2} \subset \mathbb{R}_{+}^{*}$ be some open intervals such that $I_{1} \times\{0\}$ and $\{0\} \times I_{2}$ are both contained in $V$.

Lemma 3.1. Let $\left(x_{*}, y_{*}\right) \in\left(I_{1} \times \mathbb{R}\right) \cap V$ and $\bar{t}_{I_{2}}\left(x_{*}, y_{*}\right)=\inf \{t>0$ : $\left.\phi_{J \nabla Q_{\varepsilon}}^{t}\left(x_{*}, y_{*}\right) \in\left(\mathbb{R} \times I_{2}\right) \cap V\right\}$. Then:

(1) There exists $c\left(I_{1}, I_{2}\right) \geqslant 1$ such that if $0<x_{*} y_{*} \lesssim_{I_{1}, I_{2}, \lambda} 1$ one has

$$
c\left(I_{1}, I_{2}\right)^{-1} \frac{\left|\ln \left(x_{*} y_{*}\right)\right|}{\lambda} \leqslant \bar{t}_{I_{2}}\left(x_{*}, y_{*}\right) \leqslant c\left(I_{1}, I_{2}\right) \frac{\left|\ln \left(x_{*} y_{*}\right)\right|}{\lambda} .
$$

(2) For any $(x, y)$ in a neighborhood of $\left(x_{*}, y_{*}\right)$ and any $t$ in a neighborhood of $\bar{t}_{I_{2}}\left(x_{*}, y_{*}\right)$

$$
\Xi_{2} \circ \phi_{J \nabla Q_{\varepsilon}}^{t} \circ \Xi_{1}^{-1}:(u, v) \mapsto\left(u+\tau_{\varepsilon}^{t}(v), v\right)
$$

with

$$
\tau_{\varepsilon}^{t}(v)=t q_{\varepsilon}^{\prime}(v)-\ln v
$$

Proof. 1) We evaluate $\bar{t}_{I_{2}}\left(x_{*}, y_{*}\right)$. Since

$$
\phi_{J \nabla Q_{\varepsilon}}^{t}\left(x_{*}, y_{*}\right)=\left(e^{-t q_{\varepsilon}^{\prime}\left(x_{*} y_{*}\right)} x_{*}, e^{t q_{\varepsilon}^{\prime}\left(x_{*} y_{*}\right)} y_{*}\right)
$$

we have $e^{t q_{\varepsilon}^{\prime}\left(x_{*} y_{*}\right)} y_{*} \in I_{2}$ if and only if

$$
t \in] \frac{\ln \left(\left(x_{*} y_{*}\right)^{-1} \times x_{*} \min I_{2}\right)}{q_{\varepsilon}^{\prime}\left(x_{*} y_{*}\right)}, \frac{\ln \left(\left(x_{*} y_{*}\right)^{-1} \times x_{*} \max I_{2}\right)}{q_{\varepsilon}^{\prime}\left(x_{*} y_{*}\right)}[.
$$

Hence for $x_{*} y_{*}$ small enough

$$
\left|\bar{t}_{I_{2}}\left(x_{*}, y_{*}\right)-\frac{\left|\ln \left(x_{*} y_{*}\right)\right|}{q_{\varepsilon}^{\prime}\left(x_{*} y_{*}\right)}\right| \leqslant \frac{\max \left(\left|\ln \left(x_{*} \min I_{2}\right)\right|,\left|\ln \left(x_{*} \max I_{2}\right)\right|\right)}{q_{\varepsilon}^{\prime}\left(x_{*} y_{*}\right)} .
$$

Since for $0<x_{*} y_{*} \lesssim 1$ one has $q_{\varepsilon}^{\prime}\left(x_{*} y_{*}\right) \asymp \lambda$, there exists $c\left(I_{1}, I_{2}\right)$ such that if $x_{*} y_{*}$ small enough (how small depending on $\left.I_{1}, I_{2}, \lambda\right)$ the inequality (3.26) is satisfied.

2) We write

$$
\Xi_{2} \circ \phi_{J \nabla Q_{\varepsilon}}^{t} \circ \Xi_{1}^{-1}=\Xi_{2} \circ \Xi_{1}^{-1} \circ \Xi_{1} \circ \phi_{J \nabla Q_{\varepsilon}}^{t} \circ \Xi_{1}^{-1}=\Xi_{2} \circ \Xi_{1}^{-1} \circ \phi_{J \nabla \tilde{Q}_{\varepsilon}}^{t}
$$

with $\tilde{Q}_{\varepsilon}(u, v)=\left(Q_{\varepsilon} \circ \Xi_{1}^{-1}\right)(u, v)=q_{\varepsilon}(v)$. Since $\phi_{J \nabla \tilde{Q}_{\varepsilon}}^{t}(u, v)=\left(u-t q_{\varepsilon}^{\prime}(v), v\right)$ and $\Xi_{2} \circ \Xi_{1}^{-1}(u, v)=(u-\ln v, v)$ we get (3.28) . 


\section{FUndamental DOMAINS AND FIRST RETURN MAPS}

We construct in this Section adapted fundamental domains $\mathcal{F}_{\varepsilon, y_{*}}$ for the maps $\left(f_{\varepsilon}\right)_{\varepsilon}$ satisfying conditions $(H 1)$, (H2), (H3), (H4) of Theorem 2.1 and define their first return maps $\hat{f}_{\varepsilon}$ in $\mathcal{F}_{\varepsilon, y_{*}}$.

4.1. Fundamental domains. Let $V$ be the domain of Theorem 2.1. One can choose $x_{*}>0$ such that for any $\varepsilon$ small enough

$$
\left(x_{*}, 0\right) \in V \quad \text { and } \quad f_{\varepsilon}^{-1}\left(x_{*}, 0\right) \notin V .
$$

For $y_{*}>0$ small enough, we define the vertical segment

$$
L_{x_{*}, y_{*}}:=\left\{\left(x_{*}, t y_{*}\right), 0<t<1\right\}
$$

and the domain

$$
\mathcal{F}_{\varepsilon, x *, y_{*}}
$$

as the interior of the contour defined by (see Figure 2)

(a): the segment $\left[f_{\varepsilon}\left(x_{*}, 0\right),\left(x_{*}, 0\right)\right]$,

(b): the transversal $\overline{L_{x_{*}, y_{*}}}$

(c): the piece of hyperbola joining $\left(x_{*}, y_{*}\right)$ to $f_{\varepsilon}\left(x_{*}, y_{*}\right)$ (cf. Remark 2.1)

(d): the curve $f_{\varepsilon}\left(\overline{L_{x *, y_{*}}}\right)$.

We shall often drop the index $x_{*}$ in the notations of $L_{x_{*}, y_{*}}, \mathcal{F}_{\varepsilon, x_{*}, y_{*}}$ and simply set

$$
L_{y_{*}}:=L_{x_{*}, y_{*}} \quad \text { and } \quad \mathcal{F}_{\varepsilon, y_{*}}=\mathcal{F}_{\varepsilon, x_{*}, y_{*}} .
$$

If $y_{*}$ is small enough one has $\mathcal{F}_{\varepsilon, y_{*}}, \overline{L_{y_{*}}} \subset V$. We set

$$
\tilde{\mathcal{F}}_{\varepsilon, y_{*}}=\mathcal{F}_{\varepsilon, y_{*}} \cup L_{y_{*}} .
$$

4.2. First return maps. Our aim in this subsection is to define the first return map of $f_{\varepsilon}$ in $\tilde{\mathcal{F}}_{\varepsilon, y_{*}}$.

Since $\Sigma_{\varepsilon}$ is a separatrix for $f_{\varepsilon}$ we can define (see Remark 2.2)

$$
\left.\left.N(\varepsilon) \underset{\text { defin. }}{=} \min \left\{n \in \mathbb{N}^{*}, f_{\varepsilon}^{-n}(] f_{\varepsilon}\left(x_{*}\right), x_{*}\right]\right) \subset V\right\} .
$$

We note that if $\varepsilon$ is small enough $N(\varepsilon)$ is independent of $\varepsilon$, so we shall denote it by $N$. Moreover, if $\varepsilon$ and $y_{*}$ are small enough

$$
N_{\text {defin. }}^{=} \min \left\{n \in \mathbb{N}^{*}, f_{\varepsilon}^{-n}\left(\tilde{\mathcal{F}}_{\varepsilon, y_{*}}\right) \subset V\right\} .
$$

Lemma 4.1. There exists a constant $0<c_{*}<1$ such that for $(x, y) \in \mathcal{F}_{\varepsilon, c_{*} y_{*}}$

$$
\tilde{n}_{\varepsilon}(x, y): \underset{\text { defin. }}{=} \min \left\{j \in \mathbb{N}^{*}, f_{\varepsilon}^{j}(x, y) \in f_{\varepsilon}^{-N}\left(\tilde{\mathcal{F}}_{\varepsilon, y_{*}}\right)\right\}<\infty .
$$

One has

$$
\tilde{n}_{\varepsilon}(x, y)=\ln (x y) / \lambda \text {. }
$$

Proof. Note that the domain $f_{\varepsilon}^{-N}\left(\mathcal{F}_{\varepsilon, y_{*}}\right) \subset \tilde{V}$ is the interior of the contour defined by:

(a): the segment $\left[f_{\varepsilon}^{-(N-1)}\left(x_{*}, 0\right), f_{\varepsilon}^{-N}\left(x_{*}, 0\right)\right] \subset W_{f_{\varepsilon}}^{u}(o) \cap V \subset\{0\} \times \mathbb{R}$ 
(b): the curve $f_{\varepsilon}^{-N}\left(\overline{L_{y_{*}}}\right)$

(c): a curve joining $f_{\varepsilon}^{-N}\left(x_{*}, y_{*}\right)$ to $f_{\varepsilon}^{-(N-1)}\left(x_{*}, y_{*}\right)$

(d): the curve $f_{\varepsilon}^{-(N-1)}\left(\overline{L_{y_{*}}}\right)$

and

$$
f_{\varepsilon}^{-N}\left(\tilde{\mathcal{F}}_{\varepsilon, y_{*}}\right)=f_{\varepsilon}^{-N}\left(\mathcal{F}_{\varepsilon, y_{*}}\right) \cup f_{\varepsilon}^{-N}\left(L_{y_{*}}\right) .
$$

Note that the lines $f_{\varepsilon}^{-N}\left(\overline{L_{y_{*}}}\right), f_{\varepsilon}^{-(N-1)}\left(\overline{L_{y_{*}}}\right)$ are transversal to the segment $\left[f_{\varepsilon}^{-(N-1)}\left(x_{*}, 0\right), f_{\varepsilon}^{-N}\left(x_{*}, 0\right)\right]$.

Now let $(x, y) \in \tilde{\mathcal{F}}_{\varepsilon, y_{*}}$. We denote by $\mathcal{H}_{x, y}$ the hyperbola

$$
\mathcal{H}_{x, y}:=\left\{\left(x^{\prime}, y^{\prime}\right), x^{\prime} y^{\prime}=x y\right\}
$$

and if $z, z^{\prime} \in \mathcal{H}_{x, y}$, by $\mathcal{H}_{x, y}\left(z, z^{\prime}\right)$ the arc of hyperbola of $\mathcal{H}_{x, y}$ between $z$ and $z^{\prime}$ which is open in $z$ and closed in $z^{\prime}$. If $y>0$ is small enough, $\mathcal{H}_{x, y}$ intersects $f_{\varepsilon}^{-N}\left(\tilde{\mathcal{F}}_{\varepsilon, y_{*}}\right) \subset V$ in an arc of hyperbola of the form $\mathcal{H}_{x, y}\left(p, f_{\varepsilon}^{-1}(p)\right)$ with $p \in f_{\varepsilon}^{-(N-1)}\left(L_{y_{*}}\right)$ and $f_{\varepsilon}^{-1}(p) \in f_{\varepsilon}^{-N}\left(L_{y_{*}}\right)$. The sets $f_{\varepsilon}^{-j}\left(\mathcal{H}_{x, y}\left(p, f_{\varepsilon}^{-1}(p)\right)\right.$, $j \geqslant 0$ form a partition of the semi-arc of parabola $\bigcup_{n \geqslant 0} \mathcal{H}_{x, y}\left(p, f_{\varepsilon, k}^{-n}(p)\right.$ which contains $(x, y)$. In particular, there exists $j \geqslant 0$ (in fact $j \geqslant 1$ ) such that $(x, y) \in f_{\varepsilon}^{-j}\left(\mathcal{H}_{x, y}\left(p, f_{\varepsilon}^{-1}(p)\right)\right.$ or equivalently

$$
f_{\varepsilon}^{j}(x, y) \in \mathcal{H}_{x, y}\left(p, f_{\varepsilon}^{-1}(p)\right) \subset f_{\varepsilon}^{-N}\left(\tilde{\mathcal{F}}_{\varepsilon, y_{*}}\right) .
$$

This proves (4.30).

To prove (4.31) we note that there exists an interval $I_{2}$ not containing 0 and depending only on $x_{*}, y_{*}$ such that $f_{\varepsilon}^{-N}\left(\tilde{\mathcal{F}}_{\varepsilon, y_{*}}\right) \subset \mathbb{R} \times I_{2}$. We then use Lemma 3.1 and the fact that $\left|\bar{t}_{I_{2}}(x, y)-\tilde{n}_{\varepsilon}(x, y)\right| \leqslant 1$.

We now define

$$
n_{\varepsilon}=N+\tilde{n}_{\varepsilon}
$$

By (4.31) one has

$$
n_{\varepsilon}(x, y)=\ln (x y) / \lambda .
$$

The map $\hat{f}_{\varepsilon}: \tilde{\mathcal{F}}_{\varepsilon, c_{*} y_{*}} \rightarrow \tilde{\mathcal{F}}_{\varepsilon, y_{*}}$ defined by

$$
\hat{f}_{\varepsilon}=f_{\varepsilon}^{n_{\varepsilon}}
$$

is the first return map of $f_{\varepsilon}$ in $\tilde{\mathcal{F}}_{\varepsilon, y_{*}}$ (for points starting in $\tilde{\mathcal{F}}_{\varepsilon, c_{*} y_{*}}$ ). Note that $\hat{f}_{\varepsilon}$ is not $C^{k}$ on the whole domain $\tilde{\mathcal{F}}_{\varepsilon, c_{*} y_{*}}$.

4.3. Estimates on first return maps. We denote for $a \in \mathbb{R}$

$$
T_{a}:(u, v) \mapsto(u+a, v)
$$

and we recall the definition (3.25) of the symplectic diffeomorphisms $\Xi_{1}, \Xi_{2}$.

We observe that there exist open sets $W_{1} \subset \mathbb{R}_{+}^{*} \cap \mathbb{R}, W_{2} \subset \mathbb{R} \times \mathbb{R}_{+}^{*}$ such that for any $\varepsilon$ and $y_{*}>0$ small enough

$$
\tilde{\mathcal{F}}_{\varepsilon, y_{*}} \subset W_{1} \subset V, \quad f_{0}^{-N}\left(\tilde{\mathcal{F}}_{\varepsilon, y_{*}}\right) \subset W_{2} \subset V .
$$




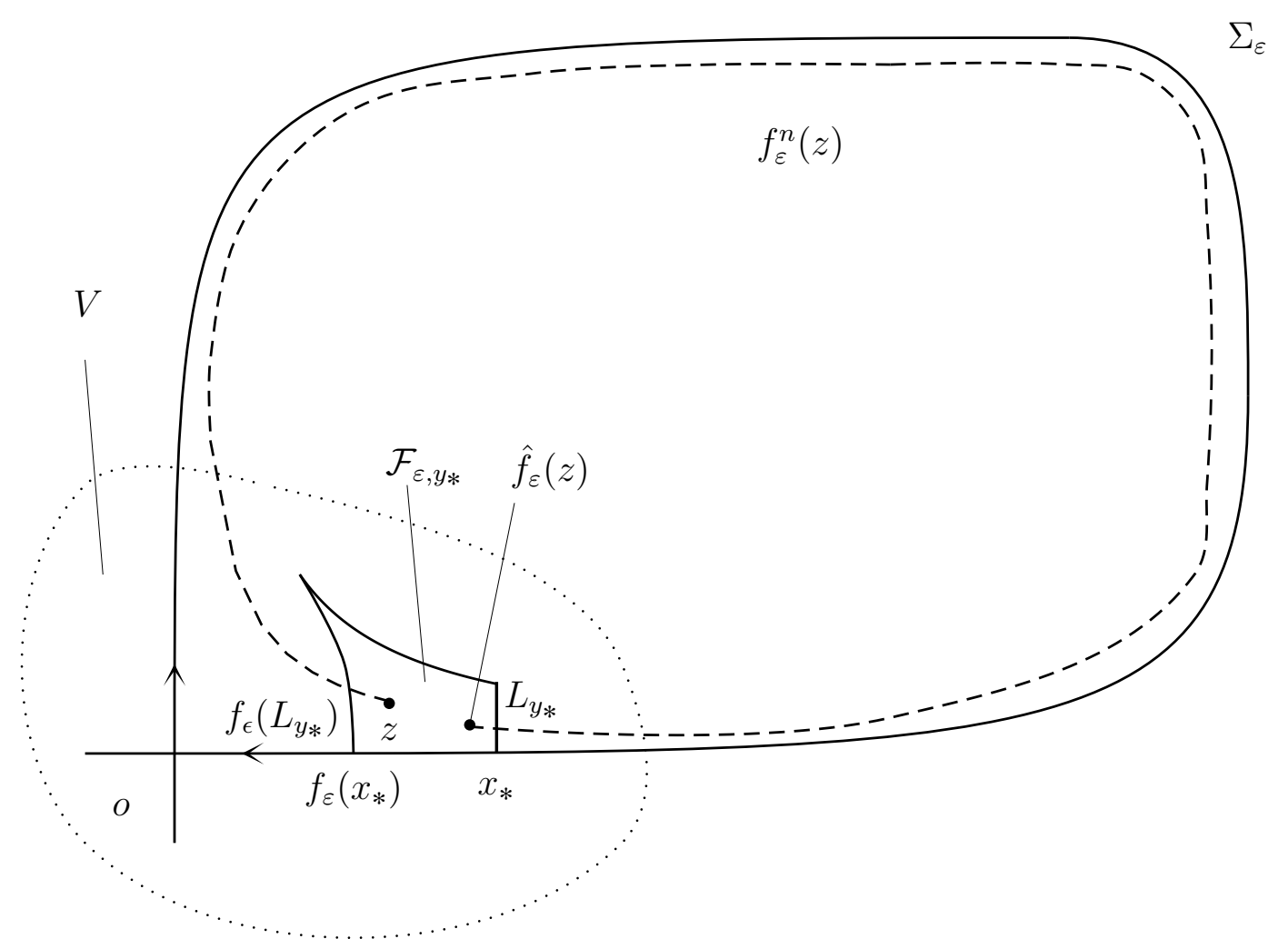

Figure 2. Fundamental domain $\mathcal{F}_{\varepsilon, y_{*}}$ for $f_{\varepsilon}$ and the first return map $\hat{f}_{\varepsilon}$.

Lemma 4.2. There exists a $C^{k}$ function $\sigma_{0, N} \in C^{k}\left(\mathbb{R}_{+}^{*}, \mathbb{R}\right)$ such that on $\Xi_{2}\left(W_{2}\right)$ one has

$$
\Xi_{1} \circ f_{0}^{N} \circ \Xi_{2}^{-1}=T_{\sigma_{0, N}} .
$$

Proof. From condition (H4) one can write on $\mathbb{R}^{2}$

$$
f_{0}=\phi_{J \nabla H_{0}}^{1}
$$

hence

$$
f_{0}^{N}=\phi_{J \nabla H_{0}}^{N}, \quad \text { where }\left.H_{0}\right|_{V}=Q_{0} .
$$

If $(u, v) \in \Sigma_{2}\left(W_{2}\right)$ and $(\tilde{u}, \tilde{v})=\Xi_{1}\left(f_{0}^{N}\left(\Xi_{2}^{-1}(u, v)\right)\right)$ one then has

$$
Q_{0}\left(\Xi_{1}^{-1}(\tilde{u}, \tilde{v})\right)=Q_{0}\left(f_{0}^{N}\left(\Xi_{2}^{-1}(u, v)\right)\right)=Q_{0}\left(\Xi_{2}^{-1}(u, v)\right)
$$

hence

$$
q_{0}(\tilde{v})=q_{0}(v)
$$

and thus $\tilde{v}=v$. Since the map $(u, v) \mapsto(\tilde{u}, \tilde{v})$ is symplectic, this forces $\tilde{u}=u+\tilde{\sigma}_{0, N}(v)$ for some $C^{k}$ function $\tilde{\sigma}_{0, N}$; this function can be extended as a $C^{k}$ function $\sigma_{0, N}: \mathbb{R} \rightarrow \mathbb{R}$. 
Recall the definition (4.34) of $\hat{f}_{\varepsilon}$.

Lemma 4.3. There exists a continuous family $\left(\hat{\eta}_{\varepsilon}\right)_{\varepsilon}$ of $C^{k}$ symplectic diffeomorphisms defined on $\mathbb{R}^{2}$ and a neighborhood $W$ of $f_{\varepsilon}^{-1}\left(\tilde{\mathcal{F}}_{\varepsilon, y_{*}}\right) \cup \tilde{\mathcal{F}}_{\varepsilon, y_{*}} \cup$ $f_{\varepsilon}\left(\tilde{\mathcal{F}}_{\varepsilon, y_{*}}\right)$ such that

$$
\left\{\begin{array}{l}
\lim _{\varepsilon \rightarrow 0}\left\|\hat{\eta}_{\varepsilon}-i d\right\|_{k}=0 \\
\hat{\eta}_{\varepsilon}(W \cap(\mathbb{R} \times\{0\}) \subset \mathbb{R} \times\{0\}
\end{array}\right.
$$

and on a neighborhood of $\tilde{\mathcal{F}}_{\varepsilon, c_{*} y_{*}}$ one has

$$
\Xi_{1} \circ \hat{f}_{\varepsilon} \circ \Xi_{1}^{-1}=\hat{\eta}_{\varepsilon} \circ T_{\hat{l}_{\varepsilon}}
$$

where

$$
\hat{l}_{\varepsilon}(v)=\sigma_{0, N}(v)+\hat{n}_{\varepsilon}(u, v) q_{\varepsilon}^{\prime}(v)-\ln v, \quad \text { with } \quad \hat{n}_{\varepsilon}=\tilde{n}_{\varepsilon} \circ \Xi_{1}^{-1} .
$$

Proof. We write (we use (4.34), (4.32), (H3)

$$
\begin{aligned}
\hat{f}_{\varepsilon} & =f_{\varepsilon}^{N+\tilde{n}_{\varepsilon}} \\
& =f_{\varepsilon}^{N} \circ \phi_{J \nabla Q_{\varepsilon}}^{\tilde{n}_{\varepsilon}} \\
& =\eta_{\varepsilon} \circ f_{0}^{N} \circ \phi_{J \nabla Q_{\varepsilon}}^{\tilde{n}_{\varepsilon}}
\end{aligned}
$$

with

$$
\eta_{\varepsilon}=f_{\varepsilon}^{N} \circ f_{0}^{-N}
$$

As a consequence, if we set

$$
\hat{\eta}_{\varepsilon}=\Xi_{1} \circ \eta_{\varepsilon} \circ \Xi_{1}^{-1} \quad \text { and } \quad \hat{n}_{\varepsilon}=\tilde{n}_{\varepsilon} \circ \Xi_{1}^{-1},
$$

we have using (4.35)

$$
\begin{aligned}
& \Xi_{1} \circ \hat{f}_{\varepsilon} \circ \Xi_{1}^{-1}=\hat{\eta}_{\varepsilon} \circ\left(\Xi_{1} \circ f_{0}^{N} \circ \Xi_{2}^{-1}\right) \circ\left(\Xi_{2} \circ \phi_{J \nabla Q_{\varepsilon}}^{\tilde{n}_{\varepsilon}} \circ \Xi_{2}^{-1}\right) \circ\left(\Xi_{2} \circ \Xi_{1}^{-1}\right) \\
& =\hat{\eta}_{\varepsilon} \circ T_{\sigma_{0, N}} \circ \phi_{J \nabla\left(Q_{\varepsilon} \circ \Xi_{2}^{-1}\right)}^{\tilde{n}_{\varepsilon} \circ \Xi_{1}^{-1}} \circ T_{-\ln v} \\
& =\hat{\eta}_{\varepsilon} \circ T_{\sigma_{0, N}} \circ T_{q_{\varepsilon}^{\prime}}^{\hat{n}_{\varepsilon}} \circ T_{-\ln v} \\
& =\hat{\eta}_{\varepsilon} \circ T_{\sigma_{0, N}+\hat{n}_{\varepsilon} q_{\varepsilon}^{\prime}-\ln v}
\end{aligned}
$$

which is (4.37) together with (4.38).

Note that by (4.39), (4.40), Remark 2.2 and the fact that $\mathbb{R} \ni \varepsilon \mapsto f_{\varepsilon} \in$ $C^{k}\left(V_{k}, \mathbb{R}^{2}\right)$ is continuous, one has

$$
\left\{\begin{array}{l}
\lim _{\varepsilon \rightarrow 0}\left\|\hat{\eta}_{\varepsilon}-i d\right\|_{C^{k}}=0 \\
\hat{\eta}_{\varepsilon}(W \cap(\mathbb{R} \times\{0\})) \subset \mathbb{R} \times\{0\} .
\end{array}\right.
$$




\section{RenORMALIZATiON}

We define in this section a renormalization $\bar{f}_{\varepsilon}$ of the map $f_{\varepsilon}$. The first return map $\hat{f}_{\varepsilon}$ of $f_{\varepsilon}$ in the fundamental domain $\mathcal{F}_{\varepsilon, y_{*}}$ we have constructed in the previous Section 4 is not differentiable at every point (see (4.37), (4.38 and the fact that the integer valued function $\hat{n}_{\varepsilon}$ has in general discontinuity points). On the other hand, if one glues the "vertical" boundaries of $\mathcal{F}_{\varepsilon, y_{*}}$ by $f_{\varepsilon}$ we obtain an abstract open annulus $\tilde{F}_{\varepsilon, y_{*}} / f_{\varepsilon}$ (see Subsections 5.1, 5.2) and the map $\hat{f}_{\varepsilon}$ is now $C^{k}$ on it. We can uniformize 9 this abstract annulus so that it becomes the standard (with the usual topology) open annulus $\mathbb{R} / \mathbb{Z} \times] 0, c[$ (some $c>0$ ), see Subsection 5.3, and the map $\hat{f}_{\varepsilon}$ in these new coordinates turns into a $C^{k}$ diffeomorphism $\bar{f}_{\varepsilon}$ defined on (part of) this standard annulus. This is the 10 renormalized diffeomorphism associated to $f_{\varepsilon}$.

5.1. Glueing. Let $\mathcal{F}$ be an open set of $\mathbb{R}^{2}, L$ a 1 -dimensional submanifold of $\mathbb{R}^{2}$ and $f$ an orientation preserving smooth diffeomorphism from a neighborhood of $\mathcal{F} \cup L$ to a neighborhood of $f(\mathcal{F} \cup L)$. We assume that:

(1) $f(\mathcal{F} \cup L) \cap(\mathcal{F} \cup L)=\varnothing$;

(2) $\mathcal{F} \cup L$ is a 2-dimensional submanifold of $\mathbb{R}^{2}$ with boundary and this boundary is $\partial(\mathcal{F} \cup L)=L$; in particular, for any point $p \in L$ there exists an open set $U_{p}, p \in U_{p} \subset \mathbb{R}^{2}$, and a smooth diffeomorphism $\varphi_{p}: U_{p} \rightarrow \varphi_{p}\left(U_{p}\right) \subset \mathbb{R}^{2}$ such that $\varphi_{p}\left(U_{p} \cap L\right)=\varphi_{p}\left(U_{p}\right) \cap(\mathbb{R} \times\{0\})$ and $\varphi_{p}\left(U_{p} \cap \mathcal{F}\right)=\varphi_{p}\left(U_{p}\right) \cap\left(\mathbb{R} \times \mathbb{R}_{+}^{*}\right)$;

(3) for any $p \in \mathcal{F} \cup L$ and $U_{p}$, one has $U_{p} \cap f(\mathcal{F} \cup L)=\varnothing$;

(4) for any $p \in L$ one has $f^{-1}\left(f\left(U_{p}\right) \cap \mathcal{F}\right)=\varphi_{p}^{-1}\left(\varphi_{p}\left(U_{p}\right) \cap\left(\mathbb{R} \times \mathbb{R}_{-}^{*}\right)\right)$, for any of the previous chart $\left(U_{p}, \varphi_{p}\right)$ at $p$.

We define the topological space $(\mathcal{F} \cup L, \mathcal{T})$ as being the set $\mathcal{F} \cup L$ endowed with the following topology $\mathcal{T}$ : a subset $S$ of $\mathcal{F} \cup L$ is an element of $\mathcal{T}$ (i.e. an open set) if for every $p \in S$ there exists an open set $V \subset \mathbb{R}^{2}$ (contained in a neighborhood of $\mathcal{F} \cup L$ where $f$ is defined) such that $V \cap f(\mathcal{F} \cup L)=\varnothing$ and $p \in(V \cup f(V)) \cap(\mathcal{F} \cup L) \subset S$.

We can then define the following differentiable structure on $(\mathcal{F} \cup L, \mathcal{T})$ as follows; (a): if $p \in \mathcal{F}$ we define the local chart $C_{p}:=\left(W_{p}, i d\right)$ where $W_{p}$ is an open set of $\mathbb{R}^{2}$ such that $p \in W_{p} \subset \mathcal{F}$; and (b): if $p \in L$ we define the local chart $C_{p}:=\left(W_{p}, \psi_{p}\right)$ where $W_{p}$ is the open set of $\mathcal{F} \cup L$ (see condition (3)) $W_{p}=(\mathcal{F} \cup L) \cap\left(U_{p} \cup f\left(U_{p}\right)\right)$ (here $\left(U_{p}, \varphi_{p}\right)$ is the local chart for $p \in L$

\footnotetext{
${ }^{9}$ Uniformizing the annulus is equivalent to conjugating $f_{\varepsilon}$ to $(x, y) \mapsto(x+1, y)$ on a domain containing $\mathcal{F}_{\varepsilon, y_{*}}$. This procedure is, in a different context, the one described in [24. We shall often call the uniformization operation normalization in reference to the corresponding renormalization procedure defined for quasi-periodic cocycles, cf. [14, 6].

${ }^{10}$ One should say "a" instead of "the" since the uniformizing/normalizing procedure is not unique.
} 
as defined in (2)) and where $\psi_{p}$ is defined by (we use condition (44)),

$$
\left\{\begin{array}{l}
\psi_{p}=\varphi_{p} \quad \text { on } \quad U_{p} \cap(\mathcal{F} \cup L)=\varphi_{p}^{-1}\left(\left(\varphi_{p}\left(U_{p}\right) \cap\left(\mathbb{R} \times \mathbb{R}_{+}\right)\right)\right. \\
\psi_{p}=\varphi_{p} \circ f^{-1} \quad \text { on } \quad f\left(U_{p}\right) \cap \mathcal{F}=f \circ \varphi_{p}^{-1}\left(\left(\varphi_{p}\left(U_{p}\right) \cap\left(\mathbb{R} \times \mathbb{R}_{-}^{*}\right)\right) .\right.
\end{array}\right.
$$

We denote by $\mathcal{A}$ the collection of all these local charts $C_{p}$ and we set $(\mathcal{F} \cup$ $L) / f=(\mathcal{F} \cup L, \mathcal{T}, \mathcal{A})$.

Remark 5.1. If we assume in addition that $f$ preserves the standard symplectic form $d x \wedge d y$ on $\mathbb{R}^{2}$, we can endow $(\mathcal{F} \cup L) / f$ with a symplectic form $\omega$.

Remark 5.2. If $g: \mathcal{F} \rightarrow g(\mathcal{F})$ is a smooth diffeomorphism defined in a neighborhood of $\mathcal{F}$, it induces a smooth diffeomorphism (that we still denote g) $g:(\mathcal{F} \cup L) / f \rightarrow(g(\mathcal{F}) \cup g(L)) /\left(g \circ f \circ g^{-1}\right)$.

Remark 5.3. If $\mathcal{F}=\left[0,1[\times] 0,1[, L=] 0,1\left[\right.\right.$ and $f=T_{1}:(x, y) \mapsto(x+1, y)$ one sees that $(\mathcal{F} \cup L) / T_{1}$ is (diffeomorphic to) the standard open annulus $(\mathbb{R} / \mathbb{Z} \times] 0,1[$, can. $)$ endowed with its canonical differentiable structure.

5.2. The space $\left(\mathcal{F}_{\varepsilon, y_{*}} \cup L_{y_{*}}\right) / f_{\varepsilon}$. If $\varepsilon$ and $y_{*}$ are small enough item (11) is satisfied and we can find charts $\left(p, U_{p}\right)$ such that items (2), (3) (41) are satisfied. See Figure 3. We can then define the manifold $\left(\mathcal{F}_{\varepsilon, y_{*}} \cup L_{y_{*}}\right) / f_{\varepsilon}$. We shall see that it is an annulus without boundary, $c f$. Lemma 5.3.

Note that if $0<c_{*}<1$, the smaller set $\tilde{\mathcal{F}}_{\varepsilon, c_{*} y_{*}}=\mathcal{F}_{\varepsilon, c_{*} y_{*}} \cup L_{c_{*} y_{*}}$ is an open subset of $\left(\mathcal{F}_{\varepsilon, y_{*}} \cup L_{y_{*}}\right) / f_{\varepsilon}$ (which means that it belongs to $\mathcal{T}$ ) and it can be endowed with the topology and differentiable structure induced by the inclusion. We denote $\left(\mathcal{F}_{\varepsilon, c_{*} y_{*}} \cup L_{c_{*} y_{*}}\right) / f_{\varepsilon}$ the thus obtained submanifold of $\left(\mathcal{F}_{\varepsilon, y_{*}} \cup L_{y_{*}}\right) / f_{\varepsilon}$. The following lemma is then tautological

Lemma 5.1. The map $\hat{f}_{\varepsilon}$ induces a $C^{k} \operatorname{map} \tilde{\mathcal{F}}_{\varepsilon, c_{*} y_{*}} / f_{\varepsilon} \rightarrow \tilde{\mathcal{F}}_{\varepsilon, y_{*}} / f_{\varepsilon}$.

We shall need in Section 6 the following lemma:

Lemma 5.2. There exists a probability measure with positive density $\pi_{\varepsilon, y_{*}}$ on $\tilde{\mathcal{F}}_{\varepsilon, y_{*}} / f_{\varepsilon}$ which is $\hat{f}_{\varepsilon}$ invariant: for any measurable set $A \in \tilde{\mathcal{F}}_{\varepsilon, y_{*}} / f_{\varepsilon}$ such that $\hat{f}_{\varepsilon}^{-1}(A) \in \tilde{\mathcal{F}}_{\varepsilon, y_{*}} / f_{\varepsilon}$ one has $\pi_{\varepsilon, y_{*}}(A)=\pi_{\varepsilon, y_{*}}\left(\hat{f}_{\varepsilon}^{-1}(A)\right)$.

Proof. We shall in fact construct this measure $\pi_{\varepsilon, y_{*}}$ on the bigger set $\hat{\mathcal{F}}_{\varepsilon, y_{*}} / f_{\varepsilon}$

$$
\hat{\mathcal{F}}_{\varepsilon, y_{*}}=\tilde{\mathcal{F}}_{\varepsilon, y_{*}} \cup \sigma\left(\tilde{\mathcal{F}}_{\varepsilon, y_{*}}\right)
$$

where $\sigma: \mathbb{R}^{2} \rightarrow \mathbb{R}^{2}$ is the reflection $(x, y) \mapsto(x,-y)$ (it commutes with $f_{\varepsilon}$ in $V$, see condition (H3)]. From Remark 5.1 there exists a symplectic form $\omega_{\varepsilon}$ on $\hat{\mathcal{F}}_{\varepsilon, y_{*}} / f_{\varepsilon}$. Note that the first return map $\hat{f}_{\varepsilon}$ is not defined on the whole set $\hat{\mathcal{F}}_{\varepsilon, y_{*}} / f_{\varepsilon}$ but nevertheless

$$
\left(\hat{f}_{\varepsilon}\right)^{*} \omega_{\varepsilon}=\omega_{\varepsilon}
$$




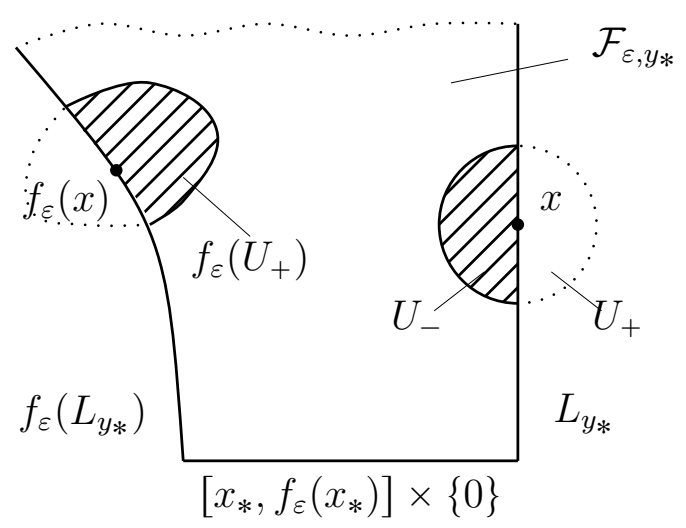

FIGURE 3. Glueing: $\left(\mathcal{F}_{\varepsilon, y_{*}} \cup L_{y_{*}}\right) / f_{\varepsilon}$

whenever this formula makes sense. The probability measure $\pi_{\varepsilon, y_{*}}$ defined by

$$
\pi_{\varepsilon, y_{*}}(A)=\int_{A}\left|\omega_{\varepsilon}\right| / \int_{\mathcal{F}_{\varepsilon, y *}}\left|\omega_{\varepsilon}\right|
$$

is $\hat{f}_{\varepsilon}$ invariant.

5.3. Normalization of $f_{\varepsilon}$. We now uniformize the abstract annulus $\tilde{\mathcal{F}}_{\varepsilon, y_{*}} / f_{\varepsilon}$. To do that it is enough to normalize $f_{\varepsilon}$ in the sense of Item 2 of the following lemma.

Lemma 5.3. [Normalization Lemma] There exists a continuous family $\left(h_{\varepsilon}\right)_{\varepsilon}$ of (not necessarily symplectic) $C^{k}$-diffeomorphisms defined on a neighborhood of $\tilde{\mathcal{F}}_{\varepsilon, y_{*}}$ such that for some $c>0$

(1) $h_{\varepsilon}$ sends $\tilde{\mathcal{F}}_{\varepsilon, y_{*}} / f_{\varepsilon}$ to the standard open annulus $((\mathbb{R} / \mathbb{Z}) \times] 0, c[$, can. $)$

(2) $h_{\varepsilon} \circ f_{\varepsilon} \circ h_{\varepsilon}^{-1}=T_{1}:(x, y) \mapsto(x+1, y)$

(3) $h_{\varepsilon}\left(\left[x_{*}, f_{\varepsilon}\left(x_{*}\right)\right] \times\{0\}=[0,1[\times\{0\}\right.$.

Proof. Using condition (H3) and the change of coordinates (3.25) of Section 3, we see that on a neighborhood of $\tilde{\mathcal{F}}_{\varepsilon, y_{*}}$ one has (we use the notation $(x, y)$ for $(u, v))$

$$
\Xi_{1} \circ f_{\varepsilon} \circ \Xi_{1}^{-1}=T_{q_{\varepsilon}^{\prime}}:(x, y) \mapsto\left(x+q_{\varepsilon}^{\prime}(y), y\right) .
$$

If $g_{\varepsilon}$ is the (not necessarily symplectic) smooth diffeomorphism

$$
g_{\varepsilon}:(x, y) \mapsto\left(\frac{x}{q_{\varepsilon}^{\prime}(y)}, y\right)
$$

one has

$$
g_{\varepsilon} \circ \Xi_{1} \circ f_{\varepsilon} \circ \Xi_{1}^{-1} \circ g_{\varepsilon}^{-1}=T_{1} .
$$


The set $\overline{\left(g_{\varepsilon} \circ \Xi_{1}\right)\left(\mathcal{F}_{\varepsilon, y_{*}}\right)}$ is of the form

$$
\overline{\left(g_{\varepsilon} \circ \Xi_{1}\right)\left(\mathcal{F}_{\varepsilon, y_{*}}\right)}=\left\{(x, y), y \in[0, c], \gamma_{\varepsilon}(y) \leqslant x \leqslant \gamma_{\varepsilon}(y)+1\right\}
$$

where $c>0, \gamma_{\varepsilon}:[0, c] \rightarrow \mathbb{R}_{+}$is $C^{k}, \gamma_{\varepsilon}(0)=0$ and the map $\mathbb{R} \ni \varepsilon \mapsto$ $\gamma_{\varepsilon} \in C^{k}([0, c], \mathbb{R})$ is continuous. This indeed follows from the definition of $\tilde{\mathcal{F}}_{\varepsilon, y_{*}}$ in subsection 4.1), the definition of $\Xi_{1}$ (3.25) and (5.41), (5.42). As a consequence, if we denote

$$
j_{\varepsilon}:(x, y) \mapsto\left(x-\gamma_{\varepsilon}(y), y\right)
$$

we have

$$
\left\{\begin{array}{l}
j_{\varepsilon} \circ T_{1}=T_{1} \circ j_{\varepsilon} \\
\left.j_{\varepsilon}\left(\left(g_{\varepsilon} \circ \Sigma_{1}\right)\left(\mathcal{F}_{\varepsilon, y_{*}}\right)\right)=\right] 0,1[\times] 0, c[ \\
\left.j_{\varepsilon}\left(\left(g_{\varepsilon} \circ \Sigma_{1}\right)\left(L_{y_{*}}\right)\right)=\{0\} \times\right] 0, c[.
\end{array}\right.
$$

By Remarks 5.2, 5.3, the map

$$
h_{\varepsilon}=j_{\varepsilon} \circ g_{\varepsilon} \circ \Xi_{1}
$$

is a diffeomorphism that sends $\tilde{\mathcal{F}}_{\varepsilon, y_{*}} / f_{\varepsilon}$ to the standard annulus $\left(\left[0,1[\times] 0, c[) / T_{1} \simeq\right.\right.$ $(\mathbb{R} / \mathbb{Z}) \times] 0, c[$ and such that

$$
h_{\varepsilon} \circ f_{\varepsilon} \circ h_{\varepsilon}^{-1}=T_{1} .
$$

To conclude the proof, we notice (2) is an immediate consequence of the definition (5.45) of $h_{\varepsilon}$.

Remark 5.4. Note that if $T_{a}(x, y)=(x+a(y), y)$ one has

$$
\left(h_{\varepsilon} \circ \Xi_{1}^{-1}\right) \circ T_{a} \circ\left(h_{\varepsilon} \circ \Xi_{1}^{-1}\right)^{-1}=T_{\tilde{a}}, \quad \tilde{a}(y)=a(y) / q_{\varepsilon}^{\prime}(y) .
$$

5.4. The renormalization $\bar{f}_{\varepsilon}$ of $f_{\varepsilon}$. There exists $\left.\delta \in\right] 0, c[$ such that the map

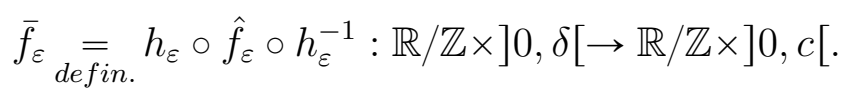

is well defined and is a $C^{k}$ diffeomorphism onto its image.

Proposition 5.4. One has

$$
\bar{f}_{\varepsilon}=\bar{\eta}_{\varepsilon} \circ T_{l_{\varepsilon}}
$$

where $\bar{\eta}_{\varepsilon}$ is a $C^{k}$ diffeomorphism defined on $\left.\mathbb{R} / \mathbb{Z} \times\right] 0, \delta\left[\right.$ and $l_{\varepsilon} \in C^{k}(] 0, c[, \mathbb{R} / \mathbb{Z})$; they satisfy

$$
\begin{gathered}
l_{\varepsilon}(y)=\frac{\sigma_{0, N}(y)}{q_{\varepsilon}^{\prime}(y)}-\frac{\ln y}{q_{\varepsilon}^{\prime}(y)} \bmod \mathbb{Z} \\
\lim _{\varepsilon \rightarrow 0}\left\|\bar{\eta}_{\varepsilon}-i d\right\|_{C^{k}}=0 \\
\bar{\eta}_{\varepsilon}:(x, y) \mapsto\left(x+a_{\varepsilon}(x, y), y+y b_{\varepsilon}(x, y)\right)
\end{gathered}
$$


where $a_{\varepsilon} \in C^{k}, b_{\varepsilon} \in C^{k-1}$ are functions defined on $\mathbb{R} / \mathbb{Z} \times(0, \delta)$.

Moreover, the map $\bar{f}_{\varepsilon}$ preserves a probability measure $\bar{\pi}_{\varepsilon, y_{*}}$ with positive density defined on $\mathbb{R} / \mathbb{Z} \times] 0, c[$.

Proof. By (4.37) and Remark 5.4 after Lemma 5.3

$$
\begin{aligned}
\bar{f}_{\varepsilon} & =\left(h_{\varepsilon} \circ \Xi_{1}^{-1}\right) \circ \hat{\eta}_{\varepsilon} \circ\left(h_{\varepsilon} \circ \Xi_{1}^{-1}\right)^{-1} \circ\left(h_{\varepsilon} \circ \Xi_{1}^{-1}\right) \circ T_{\hat{l}_{\varepsilon}} \circ\left(h_{\varepsilon} \circ \Xi_{1}^{-1}\right)^{-1} \\
& =\bar{\eta}_{\varepsilon} \circ T_{l_{\varepsilon}}
\end{aligned}
$$

where

$$
\bar{\eta}_{\varepsilon}=\left(h_{\varepsilon} \circ \Xi_{1}^{-1}\right) \circ \hat{\eta}_{\varepsilon} \circ\left(h_{\varepsilon} \circ \Xi_{1}^{-1}\right)^{-1} \quad \text { and } \quad l_{\varepsilon}(y)=\left(1 / q_{\varepsilon}^{\prime}(y)\right) \hat{l}_{\varepsilon}(y) .
$$

Since $\bar{\eta}_{\varepsilon}:=\left(h_{\varepsilon} \circ \Xi_{1}^{-1}\right) \circ \hat{\eta}_{\varepsilon} \circ\left(h_{\varepsilon} \circ \Xi_{1}^{-1}\right)^{-1}$ and $\bar{f}_{\varepsilon}$ are $C^{k}$, the function $l_{\varepsilon}$ : ] $0, c\left[\rightarrow \mathbb{R} / \mathbb{Z}\right.$ is also $C^{k}$ and

$$
l_{\varepsilon}(y)=\left(1 / q_{\varepsilon}^{\prime}(y)\right) \hat{l}_{\varepsilon}(y) .
$$

By (4.38) (remember that $\hat{n}_{\varepsilon}$ takes its value in $\mathbb{Z}$ )

$$
\begin{aligned}
l_{\varepsilon}(y) & =\frac{\sigma_{0, N}(y)}{q_{\varepsilon}^{\prime}(y)}+\hat{n}_{\varepsilon}(x, y)-\frac{\ln y}{q_{\varepsilon}^{\prime}(y)} \\
& =\frac{\sigma_{0, N}(y)}{q_{\varepsilon}^{\prime}(y)}-\frac{\ln y}{q_{\varepsilon}^{\prime}(y)} \bmod \mathbb{Z}
\end{aligned}
$$

which is (5.48).

Equation (5.49) is a consequence of the definition of $\bar{\eta}_{\varepsilon}, c f$. (5.51), the first equation of (4.36) and of the fact that $\mathbb{R} \ni \varepsilon \mapsto h_{\varepsilon} \in C^{k}$ is continuous (Lemma 5.3).

We now claim that if $\bar{\eta}_{\varepsilon}(x, y)=\left(x+a_{\varepsilon}(x, y), y+\bar{b}_{\varepsilon}(x, y)\right)$ one has for any $y$,

$$
\bar{b}_{\varepsilon}(x, 0)=0 .
$$

Indeed, since

$$
\bar{\eta}_{\varepsilon}:=\left(h_{\varepsilon} \circ \Xi_{1}^{-1}\right) \circ \hat{\eta}_{\varepsilon} \circ\left(h_{\varepsilon} \circ \Xi_{1}^{-1}\right)^{-1},
$$

equality (5.52) is a consequence of the second equation of (4.36), of item (3) of Lemma 5.3 and of the fact that $\Xi_{1}\left(\mathbb{R}_{+}^{*} \times\{0\}\right)=\mathbb{R}_{+}^{*} \times\{0\}$.

To prove (5.50) we thus notice that equality (5.52) gives us for $\bar{b}_{\varepsilon}$ a decomposition

$$
\left\{\begin{array}{l}
\bar{b}_{\varepsilon}(x, y)=y b_{\varepsilon}(x, y) \\
b_{\varepsilon} \in C^{k-1}
\end{array}\right.
$$

Finally to conclude the proof of the Proposition we observe that since the $\operatorname{map} \hat{f}_{\varepsilon}: \tilde{\mathcal{F}}_{\varepsilon, c_{*} y_{*}} / f_{\varepsilon} \rightarrow \tilde{\mathcal{F}}_{\varepsilon, y_{*}} / f_{\varepsilon}$ preserves the probability measure $\pi_{\varepsilon, y_{*}}, c f$. Lemma [5.2, the diffeomorphism $\left.\bar{f}_{\varepsilon}: \mathbb{R} / \mathbb{Z} \times\right] 0, \delta[\rightarrow \mathbb{R} / \mathbb{Z} \times] 0, c[$ preserves the probability measure $\bar{\pi}_{\varepsilon, y_{*}}=\left(h_{\varepsilon}\right)_{*} \pi_{\varepsilon, y_{*}}$ defined on $\left.\mathbb{R} / \mathbb{Z} \times\right] 0, c[$ (in the sense that if $A \subset \mathbb{R} / \mathbb{Z} \times] 0, c\left[\right.$ is a Borelian set such that $\left.\bar{f}_{\varepsilon}^{-1}(A) \subset \mathbb{R} / \mathbb{Z} \times\right] 0, c[$, one has $\left.\bar{\pi}_{\varepsilon, y_{*}}(A)=\bar{\pi}_{\varepsilon, y_{*}}\left(\bar{f}_{\varepsilon}^{-1}(A)\right)\right)$. 


\section{Applying the Translated Curve Theorem}

We apply in this Section Rüssmann's (or Moser's) Translated Curve Theorem to some rescaled version $f_{\varepsilon, n}$ of the renormalization $\bar{f}_{\varepsilon}$ of $f_{\varepsilon}$ defined in Section 5.4.

6.1. The Translated Curve Theorem. Let $\psi: \mathbb{R} / \mathbb{Z} \times] e^{-1}, 1[\rightarrow \mathbb{R} / \mathbb{Z} \times \mathbb{R}$ $(\ln e=1)$ be a $C^{k}$ diffeomorphism defined on the annulus (or cylinder) $\mathbb{R} / \mathbb{Z} \times] e^{-1}, 1\left[\right.$. We say that the graph $\operatorname{Gr}_{\gamma}:=\{(x, \gamma(x)): x \in \mathbb{R} / \mathbb{Z}\}$ of a continuous map $\gamma: \mathbb{R} / \mathbb{Z} \rightarrow \mathbb{R} / \mathbb{Z} \times] e^{-1}, 1[$ is translated by $\psi$ if for some $t \in \mathbb{R}$

$$
\psi\left(\mathrm{Gr}_{\gamma}\right)=\mathrm{Gr}_{t+\gamma}
$$

and invariant if $t=0$. If $\mathrm{Gr}_{\gamma}$ satisfies (6.53), there exists an orientation preserving homeomorphism of the circle $g: \mathbb{R} / \mathbb{Z} \rightarrow \mathbb{R} / \mathbb{Z}$ such that $\psi(x, \gamma(x))=\psi(g(x), t+\gamma(g(x))$. If $t=0$ (resp. $t \neq 0$ ), we define (resp. with a clear abuse of language) the rotation number of ( $\psi$ on) the invariant (resp. translated) graph $\mathrm{Gr}_{\gamma}$ as the rotation number of the circle diffeomorphism $g$. We say that $\psi$ has the intersection property if for any continuous $\gamma: \mathbb{R} / \mathbb{Z} \rightarrow \mathbb{R} / \mathbb{Z} \times] e^{-1}, 1\left[\right.$, the curve $\operatorname{Gr}_{\gamma}:=\{(x, \gamma(x)): x \in \mathbb{R} / \mathbb{Z}\}$ intersects its image $\psi\left(\mathrm{Gr}_{\gamma}\right)$. Note the following important fact: If $\psi$ has the intersection property, any translated graph by $\psi$ is invariant.

We state the Translated Curve Theorem by Rüssmann [17] (which implies the Invariant Curve Theorem by Moser [15]):

Theorem 6.1 (Rüssmann, [17]). There exists $k_{0} \in \mathbb{N}$ for which the following holds. Let $k \geqslant k_{0}, C, \mu>0$ and $l: \mathbb{R} / \mathbb{Z} \rightarrow \mathbb{R} a C^{k}$ map satisfying the twist condition

$$
\min _{y}\left|\partial_{y} l(y)\right|>\mu>0 \quad \text { and } \quad\|l\|_{C^{k_{0}}} \leqslant C
$$

and define

$$
\psi_{0}:(x, y) \mapsto(x+l(y), y) .
$$

There exists $\varepsilon_{0}=\varepsilon_{0}(C, \mu)>0$ such that, for any $C^{k}$ diffeomorphism

$$
\psi: \mathbb{R} / \mathbb{Z} \times] e^{-1}, 1[\rightarrow \mathbb{R} / \mathbb{Z} \times \mathbb{R}
$$

satisfying

$$
\left\|\psi-\psi_{0}\right\|_{C^{k_{0}}}<\varepsilon_{0},
$$

the diffeomorphism $\psi$ admits a set of positive Lebesgue measure of $C^{k-k_{0}}$ translated graphs contained in $(\mathbb{R} / \mathbb{Z}) \times] e^{-3 / 4}, e^{-1 / 4}[$. Moreover, all these translated graphs have Diophantine rotation number 11 .

\footnotetext{
${ }^{11}$ They are in a fixed Diophantine class $D C(\kappa, \tau)$ (the exponent is $\tau$ and the constant $\kappa)$ that can be prescribed in advance once $\mu$ is fixed ( $k_{0}$ then depends on $\tau$ and $\varepsilon_{0}$ on $\kappa$ and $\tau)$.
} 


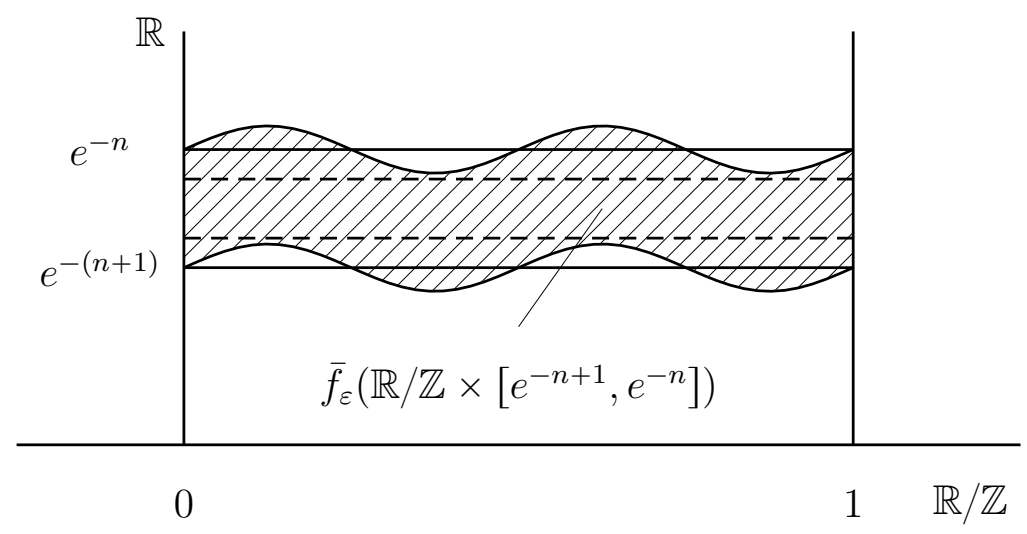

Figure 4. The diffeomorphism $\bar{f}_{\varepsilon}$ on $\mathbb{R} / \mathbb{Z} \times\left[e^{-(n+1)}, e^{-n}\right]$.

6.2. The rescaled diffeomorphism $\stackrel{\circ}{f}, n_{\varepsilon, n}$. Let $\bar{f}_{\varepsilon}$ be the renormalized map defined in Section 5.4 and define $u_{\varepsilon}, v_{\varepsilon}$ by

$$
\bar{f}_{\varepsilon}(x, y)=\left(x+u_{\varepsilon}(x, y), y+v_{\varepsilon}(x, y)\right) .
$$

Since $\bar{f}_{\varepsilon}=\bar{\eta}_{\varepsilon} \circ T_{l_{\varepsilon}}(c f$. (5.47)) one has using (5.50)

$$
\begin{aligned}
& u_{\varepsilon}(x, y)=l_{\varepsilon}(y)+a_{\varepsilon}\left(x+l_{\varepsilon}(y), y\right) \\
& v_{\varepsilon}(x, y)=y b_{\varepsilon}\left(x+l_{\varepsilon}(y), y\right) .
\end{aligned}
$$

Now, let $n \in \mathbb{N}^{*}$ large enough so that

$$
] e^{-(n+1)}, e^{-n}[\subset] 0, \delta[
$$

(the $\delta$ of (5.46) ) and introduce the rescaled $C^{k}$ diffeomorphism $\stackrel{\circ}{\varepsilon, n}_{\varepsilon}$ defined on the annulus $\mathbb{R} / \mathbb{Z} \times] e^{-1}, 1[$ by

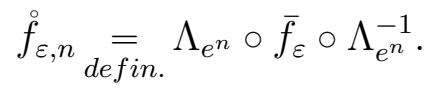

where $\Lambda_{e^{n}}:(x, y) \mapsto\left(x, e^{n} y\right)$. Let us denote

$$
\stackrel{\circ}{f, n}_{\varepsilon, n}(x, y)=\left(x+u_{\varepsilon, n}(x, y), y+v_{\varepsilon, n}(x, y)\right) .
$$

A computation shows that:

$$
\left\{\begin{array}{l}
u_{\varepsilon, n}(x, y)=l_{\varepsilon, n}(y)+a_{\varepsilon}\left(x+l_{\varepsilon, n}(y), e^{-n} y\right) \\
v_{\varepsilon, n}(x, y)=y b_{\varepsilon}\left(x+l_{\varepsilon, n}(y), e^{-n} y\right)
\end{array}\right.
$$

where

$$
l_{\varepsilon, n}(y)=l_{\varepsilon}\left(e^{-n} y\right)
$$

We can now state the following important proposition the proof of which occupies the next subsection: 
Proposition 6.1. Assume that $k \geqslant k_{0}+2$ ( $k$ is the regularity in conditions (H1) (H4) and $k_{0}$ is the one of Theorem [6.1). There exists $\varepsilon_{1}>0$ such that the following holds. If $|\varepsilon| \leqslant \varepsilon_{1}$ and $n \gg 1, \AA_{\varepsilon, n}^{\circ}$ admits a set of positive Lebesgue measure of invariant $C^{k-k_{0}-2}$-graphs in $\left.\mathbb{R} / \mathbb{Z} \times\right] e^{-1}, 1[$.

\subsection{Proof of Proposition 6.1.}

6.3.1. Twist condition for $l_{\varepsilon, n}$.

Lemma 6.2. There exist $C, \mu>0$ such that for any $\varepsilon$ small enough and any $n \gg 1$ the map $l_{\varepsilon, n}$ satisfies the twist condition (6.54) provided $k \geqslant k_{0}+1$.

Proof. Using (5.48), (6.59) we have

$$
\begin{aligned}
l_{\varepsilon, n}(y) & =l_{\varepsilon}\left(e^{-n} y\right) \\
& =\frac{\sigma_{0, N}\left(e^{-n} y\right)}{q_{\varepsilon}^{\prime}\left(e^{-n} y\right)}+\frac{n}{q_{\varepsilon}^{\prime}\left(e^{-n} y\right)}-\frac{\ln y}{q_{\varepsilon}^{\prime}\left(e^{-n} y\right)} \quad \bmod \mathbb{Z} \\
& =\frac{\sigma_{0, N}(0)+n}{\lambda}-\frac{\ln y}{\lambda}+\theta_{\varepsilon, n}(y) \quad \bmod \mathbb{Z}
\end{aligned}
$$

where

$$
\left\|\theta_{\varepsilon, n}\right\|_{C^{k-1}\left(\left[e^{-1}, 1\right]\right)}=O\left(e^{-n}\right)
$$

this last inequality is a consequence of the fact that $q_{\varepsilon}(s)=\lambda s+O\left(s^{2}\right)$ is continuous w.r.t. $\varepsilon$ ( $c f$. condition $(H 2)$ ) and of the fact that $\sigma_{0, N}$ is $C^{k}(c f$. Lemma 4.2). In particular for some $C_{k}>0$ (depending on $\lambda$ )

$$
\left\|l_{\varepsilon, n}\right\|_{C^{k-1}} \leqslant C_{k}
$$

and since $\partial_{y} l_{\varepsilon, n}(y)=-1 /(\lambda y)+\partial_{y} \theta_{\varepsilon, n}(y)$ and $\left.y \in\right] e^{-1}, 1[$

$$
\left|\partial_{y} l_{\varepsilon, n}(y)\right| \geqslant 1 /(2 \lambda) \text {. }
$$

Hence (6.54) holds uniformly in $\varepsilon, n$ with $C=C_{k_{0}+1}$ and $\mu=1 /(2 \lambda)$ as soon as $n$ is large enough.

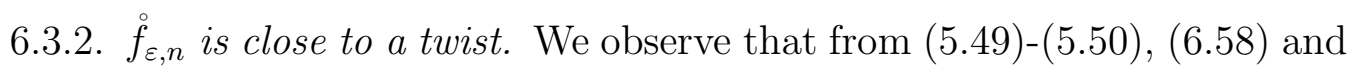
Lemma 6.2 one has uniformly in $n$

$$
\lim _{\varepsilon \rightarrow 0} \max \left(\left\|u_{\varepsilon, n}-l_{\varepsilon, n}\right\|_{C^{k-2}},\left\|v_{\varepsilon, n}\right\|_{C^{k-2}}\right)=0 .
$$

In particular, if $n$ is large enough, inequality (6.55) is satisfied if $k \geqslant k_{0}+2$ with $\psi=\stackrel{\circ}{f}_{\varepsilon, n}$ and $\psi_{0}:(x, y) \mapsto\left(x+l_{\varepsilon, n}(y), y\right)$.

We see from 6.3.1, 6.3.2 that, if

$$
|\varepsilon| \leqslant \varepsilon_{1 \text { defin. }} \varepsilon_{0}\left(C_{k_{0}+1}, 1 /(2 \lambda)\right)
$$

and $n \gg 1$, the assumptions of Theorem 6.1 are then satisfied by $\stackrel{\circ}{\varepsilon, n}$ with $k-2$ in place of $k$. Under these conditions, there thus exists a set $\mathcal{G}_{\varepsilon, n}$ of

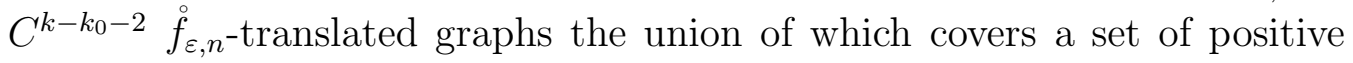


Lebesgue measure in $(\mathbb{R} / \mathbb{T}) \times] e^{-3 / 4}, e^{-1 / 4}[$. We just have to check that these translated graphs are indeed invariant.

6.3.3. $\dot{\circ}_{\varepsilon, n}$-translated graphs are invariant. Let $\left.\dot{\gamma} \subset(\mathbb{R} / \mathbb{T}) \times\right] e^{-3 / 4}, e^{-1 / 4}[$ be

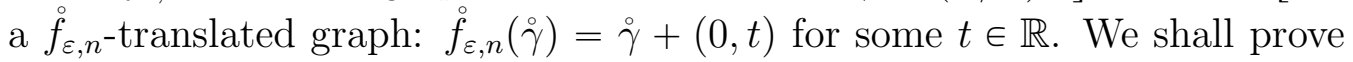
that $t=0$. We can without loss of generality assume that $t \geqslant 0$ (the case $t \leqslant 0$ is treated in a similar way).

Formula (6.60) shows that if $n \gg 1$ one has $\left.\stackrel{\circ}{\varepsilon, n}_{\varepsilon}(\stackrel{\circ}{\gamma}) \subset(\mathbb{R} / \mathbb{T}) \times\right] e^{-1}, 1[$. From the conjugation relation (6.57) we see that (cf. (6.56))

$$
\bar{\gamma}:=\Lambda_{e^{n}}^{-1}(\stackrel{\gamma}{\gamma} \subset(\mathbb{R} / \mathbb{Z}) \times] e^{-n-3 / 4}, e^{-n-1 / 4}[\subset(\mathbb{R} / \mathbb{Z}) \times] 0, \delta[
$$

is a $\bar{f}_{\varepsilon}$-translated graph such that

$$
\left.\bar{f}_{\varepsilon}(\bar{\gamma})=\bar{\gamma}+\left(0, e^{-n} t\right) \subset(\mathbb{R} / \mathbb{Z}) \times\right] e^{-(n+1)}, e^{-n}[\subset(\mathbb{R} / \mathbb{Z}) \times] 0, \delta[.
$$

Let $A$ be the open domain of $(\mathbb{R} / \mathbb{Z}) \times] 0, c[$ between $(\mathbb{R} / \mathbb{Z}) \times\{0\}$ and $\bar{\gamma}$. Since $t \geqslant 0$, one has $\left.A \subset \bar{f}_{\varepsilon}(A) \subset(\mathbb{R} / \mathbb{Z}) \times\right] 0, c[$.

Assume by contradiction that $t>0$; then the set $\bar{f}_{\varepsilon}(A) \backslash A$ contains a nonempty open set. We have seen (cf. Proposition 5.4) that $\bar{f}_{\varepsilon}$ preserves a probability measure $\bar{\pi}_{\varepsilon, y_{*}}$ with positive density defined on $\left.(\mathbb{R} / \mathbb{Z}) \times\right] 0, c[$, so $\bar{\pi}_{\varepsilon, y_{*}}\left(\bar{f}_{\varepsilon}(A) \backslash A\right)>0$. But this contradicts the invariance of $\bar{\pi}_{\varepsilon, y_{*}}$ by $\bar{f}_{\varepsilon}$.

The proof of Proposition 6.1 is complete.

6.4. Invariant curves for $\bar{f}_{\varepsilon}$. We can now state:

Theorem 6.2. Let $k \geqslant k_{0}+2$ and $|\varepsilon| \leqslant \varepsilon_{1}$. There exists $\left.\nu \in\right] 0, \delta[$ such that for any $\nu \in] 0, \nu\left[\right.$ there exists a set $\overline{\mathcal{G}}_{\varepsilon, \nu}$ of $C^{k-k_{0}-2}, \bar{f}_{\varepsilon}$-invariant graphs contained in $(\mathbb{R} / \mathbb{Z}) \times] e^{-1} \nu, \nu[$ such that

$$
\operatorname{Leb}_{\mathbb{R}^{2}}\left(\bigcup_{\bar{\gamma} \in \overline{\mathcal{G}}_{\varepsilon, \nu}} \bar{\gamma}\right)>0
$$

Proof. We choose $n$ so that

$$
] e^{-(n+1)}, e^{-n}[\subset] 0, \nu[
$$

and we observe that when $\nu \rightarrow 0$ one has $n \rightarrow \infty$. Define

$$
\stackrel{\circ}{f}, n_{\varepsilon, n} \Lambda_{e^{n}} \circ \bar{f}_{\varepsilon} \circ \Lambda_{e^{n}}^{-1} \text {. }
$$

By Proposition [6.1, there exists $\nu_{1}>0$ such that if $\left.\nu \in\right] 0, \nu_{1}[(n$ satisfying (6.61) is then large enough), the diffeomorphism $f_{\varepsilon, n}^{\circ}$ admits $C^{k-k_{0}-2}$ invariant curves in $\mathbb{T} \times] e^{-1}, 1$ [ covering a set of positive Lebesgue measure; hence $\bar{f}_{\varepsilon, k}$ has $C^{k-k_{0}-2}$-invariant curves in $\left.\mathbb{T} \times\right] e^{-1} \nu, \nu[$ covering a set of positive Lebesgue measure.

We shall denote

$$
\overline{\mathcal{G}}_{\varepsilon}=\bigcup_{\nu \in] 0, \nu_{1}[} \overline{\mathcal{G}}_{\varepsilon, \nu} .
$$


Remark 6.1. For all $\bar{\gamma} \in \overline{\mathcal{G}}_{\varepsilon, \nu}$ the rotation number of the circle diffeomorphism $\left.\bar{f}_{\varepsilon}\right|_{\bar{\gamma}}$ is Diophantine in a fixed Diophantine class $D C(\kappa, \tau)$ (see Footnote 11 in the statement of Theorem 6.1).

\section{INVARIANT CURVES FOR $f_{\varepsilon}$}

We define

$$
r=k-k_{0}-2
$$

and assume that $|\varepsilon| \leqslant \varepsilon_{1}$.

Let $\bar{\gamma} \subset(\mathbb{R} / \mathbb{Z}) \times] 0, \delta\left[, \bar{\gamma} \in \overline{\mathcal{G}}_{\varepsilon}\right.$, be a $C^{r}$ invariant graph for $\left.\bar{f}_{\varepsilon}:(\mathbb{R} / \mathbb{Z}) \times\right] 0, \delta[\rightarrow$ $(\mathbb{R} / \mathbb{Z}) \times] 0, c\left[\right.$. Note that there exists $\delta_{1}>0$ such that $\left.\bar{\gamma} \subset(\mathbb{R} / \mathbb{Z}) \times\right] \delta_{1}, \delta[$.

We can view $\bar{\gamma}$ as an invariant graph sitting in $\left(\left[0,1[\times] 0, c[) / T_{1}\right.\right.$ (recall $\left.T_{1}(x, y)=(x+1, y)\right)$. In particular one can find a $C^{r}, 1$-periodic function

$$
\bar{z}: \mathbb{R} \rightarrow\left(\left[0,1[\times] 0, \delta[) / T_{1}\right.\right.
$$

such that for all $t, \frac{d}{d t} \bar{z}(t) \neq 0$ and

$$
\bar{\gamma}=\bar{z}\left([0,1[), \quad \bar{z}(0) \in\{0\} \times] 0, c\left[\quad \lim _{t \rightarrow 1-} \bar{z}(t)=T_{1}(\bar{z}(0)) \in\{1\} \times\right] 0, c[.\right.
$$

Let

$$
\hat{\gamma}=h_{\varepsilon}^{-1}(\bar{\gamma})
$$

where $h_{\varepsilon}$ was defined in Lemma 5.3. Since $\bar{f}_{\varepsilon}=h_{\varepsilon} \circ \hat{f}_{\varepsilon} \circ h_{\varepsilon}^{-1}(c f$. (5.46) $)$ we see that

$$
\hat{\gamma} \subset h_{\varepsilon}^{-1}((\mathbb{R} / \mathbb{Z}) \times] \delta_{1}, \delta[) \subset \tilde{\mathcal{F}}_{\varepsilon, c_{*} y *}
$$

is a $C^{r}$ compact, connected, 1-dimensional submanifold (without boundary) of $\tilde{\mathcal{F}}_{\varepsilon, c_{*} y_{*}} / f_{\varepsilon}$ which is invariant by $\hat{f}_{\varepsilon}: \tilde{\mathcal{F}}_{\varepsilon, c_{*} y_{*}} / f_{\varepsilon} \rightarrow \tilde{\mathcal{F}}_{\varepsilon, y_{*}} / f_{\varepsilon}$. Moreover, the function

$$
\hat{z} \underset{\text { defin. }}{=} h_{\varepsilon}^{-1} \circ \bar{z}: \mathbb{R} \rightarrow \tilde{\mathcal{F}}_{\varepsilon, c_{*} y *} / f_{\varepsilon}
$$

is a $C^{r}, 1$-periodic function and

$$
\hat{\gamma}=\hat{z}\left(\left[0,1[), \quad \hat{z}(0) \in L_{y_{*}} \quad \lim _{t \rightarrow 1-} \hat{z}(t)=f_{\varepsilon}(\hat{z}(0)) \in f_{\varepsilon}\left(L_{y_{*}}\right) .\right.\right.
$$

The main result of this section is the following proposition.

Proposition 7.1. The set

$$
\hat{\Gamma}=\bigcup_{n \in \mathbb{Z}} f_{\varepsilon}^{n}(\hat{\gamma}) \subset \mathbb{R}^{2}
$$

is an invariant $C^{r}$ curve for $f_{\varepsilon}$ : it is a compact, connected, 1-dimensional $C^{r}$ submanifold of $\mathbb{R}^{2}$ which is invariant by $f_{\varepsilon}$.

We give the proof of this proposition in subsection 7.2 . 
7.1. Preliminary results. We define the function $\hat{Z}: \mathbb{R} \rightarrow \mathbb{R}^{2}$

$$
\forall t \in \mathbb{R}, \quad \hat{Z}(t)=f_{\varepsilon, k}^{[t]}(\hat{z}(t-[t]))
$$

([t] denotes the integer part of $t$ i.e. the unique integer such that $[t] \leqslant t<$ $[t]+1)$.

Lemma 7.2. The function $\hat{Z}: \mathbb{R} \rightarrow \mathbb{R}^{2}$ is $C^{r}$.

Proof. Note that for $t \in[0,1[, \hat{Z}(t)=\hat{z}(t)$. Also, the very definition of $\tilde{\mathcal{F}}_{\varepsilon, y_{*}} / f_{\varepsilon, k}$ shows that the function $\hat{Z}$ is $C^{r}$ on a neighborhood of $t=1$. It is hence $C^{r}$ on $\left[0,2\left[\right.\right.$ and since for $j \in \mathbb{Z}, \hat{Z}(t+j)=f_{\varepsilon}^{j}(\hat{Z}(t))$, it is $C^{r}$ on $\mathbb{R}$.

Let us set

$$
\tau_{\text {defin. }}^{=} \inf \left\{t \geqslant 1, \hat{Z}(t) \in \tilde{\mathcal{F}}_{\varepsilon, y_{*}}\right\}
$$

Note that

$$
2 \leqslant \tau<\infty
$$

Indeed, the left hand side inequality is a consequence of the fact that $\tilde{\mathcal{F}}_{\varepsilon, y_{*}} \cap$ $f_{\varepsilon}\left(\tilde{\mathcal{F}}_{\varepsilon, y_{*}}\right)=\varnothing$. For the right hand side, we observe that since $\hat{Z}(0)=\hat{z}(0) \in$ $\tilde{\mathcal{F}}_{\varepsilon, c_{*} y_{*}}$, one has (see (4.34) $), \hat{Z}\left(n_{\varepsilon}(\hat{z}(0))\right)=f_{\varepsilon}^{n_{\varepsilon}(\hat{z}(0))}(\hat{z}(0)) \in \tilde{\mathcal{F}}_{\varepsilon, y_{*}}$, hence $\tau \leqslant n_{\varepsilon}(\hat{z}(0))<\infty$.

Lemma 7.3. The map $\hat{Z}:\left[0, \tau\left[\rightarrow \mathbb{R}^{2}\right.\right.$ is injective.

Proof. Assume by contradiction that $\hat{Z}:\left[0, \tau\left[\rightarrow \mathbb{R}^{2}\right.\right.$ is not injective; then, there exists $m_{i} \in \mathbb{N}, 0 \leqslant s_{i}<1$,

$$
0 \leqslant m_{i}+s_{i}<\tau, \quad i=1,2, \quad \hat{Z}\left(s_{1}+m_{1}\right)=\hat{Z}\left(s_{2}+m_{2}\right) .
$$

Hence $f_{\varepsilon}^{m_{1}}(\hat{\gamma}) \cap f_{\varepsilon}^{m_{2}}(\hat{\gamma}) \neq \varnothing$ and if $m:=m_{2}-m_{1} \geqslant 0, f_{\varepsilon}^{m}(\hat{\gamma}) \cap \hat{\gamma} \neq \varnothing$. In particular, there exists $t \in\left[m, m+1\left[\right.\right.$ such that $\hat{Z}(t) \in \tilde{\mathcal{F}}_{\varepsilon, y_{*}}$ and then $t \geqslant \tau$. As a consequence $m>\tau-1$ and since $0 \leqslant m<\tau\left(m_{1}, m_{2}\right.$ are both in the interval $\left[0, \tau[)\right.$ one has $m=[\tau]$ hence $m_{2}=m=[\tau]$ and $m_{1}=0$. We then have from (7.63), $\hat{Z}\left(s_{2}+[\tau]\right)=\hat{Z}\left(s_{1}\right) \in \tilde{\mathcal{F}}_{\varepsilon, y_{*}}$ (since $s_{1} \in[0,1[$ ) hence by the definition of $\tau, s_{2}+[\tau] \geqslant \tau$ which contradicts $m_{2}+s_{2}<\tau$.

Lemma 7.4. If for some $t \geqslant 1, \hat{Z}(t) \in \tilde{\mathcal{F}}_{\varepsilon, y_{*}}$, then $\hat{Z}(t) \in \hat{\gamma}$.

Proof. Indeed, writing $t=s+n, s \in\left[0,1\left[, n \in \mathbb{N}^{*}\right.\right.$, one has $\hat{Z}(t)=$ $f_{\varepsilon}^{n}(\hat{z}(s))$. The integer $n \geqslant 1$ is thus a $m^{\text {th }}$ return time of $\hat{z}(s)$ in $\tilde{\mathcal{F}}_{\varepsilon, y_{*}}$, $\hat{Z}(t)=\hat{f}_{\varepsilon}^{m}(\hat{z}(s))$, and since $\hat{\gamma}$ is invariant by $\hat{f}_{\varepsilon}$, it is readily seen by induction on $m$ that $f_{\varepsilon}^{n}(\hat{z}(s)) \in \hat{\gamma}$.

Lemma 7.5. One has $\hat{Z}(\tau)=\hat{z}(0)$. 
Proof. From the definition of $\tau$ and Lemma[7.4 we have $\hat{Z}(\tau) \in \operatorname{closure}(\hat{\gamma}) \cap$ $\operatorname{closure}\left(L_{y_{*}} \cup f_{\varepsilon}\left(L_{y_{*}}\right)\right)$ hence $\hat{Z}(\tau) \in\left\{\hat{z}(0), f_{\varepsilon}(\hat{z}(0))\right\}$. To conclude we observe that one cannot have $\hat{Z}(\tau)=f_{\varepsilon}(\hat{z}(0))$ since otherwise one would have $\hat{Z}(\tau-$ $1)=\hat{z}(0) \in \tilde{\mathcal{F}}_{\varepsilon, y_{*}}$ which contradicts the definition of $\tau$ (from (7.62) $\tau-1 \geqslant 1$ ).

Lemma 7.6. The derivative of $\hat{Z}$ at $\tau$ is transverse to $L_{y_{*}}$.

Proof. 1) If there exists a sequence $t_{n} \in \mathbb{R}, \lim t_{n}=\tau$ such that $Z\left(t_{n}\right) \in$ $\tilde{\mathcal{F}}_{\varepsilon, y_{*}}$, then from Lemma (7.4) one has $Z\left(t_{n}\right) \in \hat{\gamma}$ and consequently $(d \hat{Z} / d t)(\tau)$ is tangent to $\hat{\gamma}$, thus transverse to $L_{y_{*}}$.

2) Otherwise, there exists an open interval $I \subset \mathbb{R}, I \ni \tau$, such that for all $t \in I \backslash\{\tau\}, \hat{Z}(t) \notin \tilde{\mathcal{F}}_{\varepsilon, y_{*}}$ and $f_{\varepsilon}(\hat{Z}(t)) \in \mathcal{F}_{\varepsilon, y_{*}}$. From Lemma (7.4) one then has for all $t \in I \backslash\{\tau\}, \hat{Z}(t+1)=f_{\varepsilon}(\hat{Z}(t)) \in \hat{\gamma}$ (see item (2) of Subsection 5.1) hence $D f_{\varepsilon}\left(f_{\varepsilon}(\hat{Z}(\tau))\right) \cdot(d \hat{Z} / d t)(\tau)$ is tangent to $\hat{\gamma}$ and in particular transverse to $f_{\varepsilon}\left(L_{y_{*}}\right)$. This implies that $(d \hat{Z} / d t)(\tau)$ is transverse to $L_{y_{*}}$.

Lemma 7.7. One has $\hat{Z}([\tau, \tau+1[)=\hat{Z}([0,1[)$.

Proof. We define $s_{*}=\sup \left\{s \geqslant 0: \forall t \in\left[\tau, \tau+s\left[, \hat{Z}(t) \in \mathcal{F}_{\varepsilon, y_{*}}\right\}\right.\right.$. From Lemmata 7.4 and 7.6 one has: (a) $s_{*}>0$; (b) for any $t \in\left[\tau, \tau+s_{*}[\right.$, $\hat{Z}(t) \in \hat{\gamma}$; and $(\mathrm{c}) \hat{Z}\left(\tau+s_{*}\right) \in f_{\varepsilon}\left(L_{y_{*}}\right) \cap \operatorname{closure}(\hat{\gamma})=f_{\varepsilon}(\hat{z}(0))=\hat{Z}(1)$. In particular, $\hat{Z}\left(\tau+s_{*}-1\right)=f_{\varepsilon}^{-1}(\hat{Z}(1))=\hat{Z}(0) \in \tilde{\mathcal{F}}_{\varepsilon, y_{*}}$ and by the definition of $\tau$, this implies $s_{*} \geqslant 1$. Now we notice that one cannot have $s_{*}>1$ because otherwise $\tau+1 \in\left[\tau, \tau+s_{*}\left[\right.\right.$ and by definition of $s_{*}, \hat{Z}(\tau+1) \in \mathcal{F}_{\varepsilon, y_{*}}$; but $\hat{Z}(\tau+1)=f_{\varepsilon}(\hat{Z}(\tau)$ ) and since $\hat{Z}(\tau)=\hat{z}(0)$ (Lemma 7.5) one has $\hat{Z}(\tau+1)=f_{\varepsilon}(\hat{z}(0)) \notin \mathcal{F}_{\varepsilon, y_{*}}$. We have hence proven that $s_{*}=1$. This implies that $\hat{Z}([\tau, \tau+1[)=\hat{Z}([0,1[)$.

7.2. Proof of Proposition 7.1. We first observe that

$$
\hat{\Gamma}=\bigcup_{n \in \mathbb{Z}} f_{\varepsilon}^{n}(\hat{\gamma})=\hat{Z}(\mathbb{R})=\bigcup_{n \in \mathbb{Z}} f_{\varepsilon}^{n}(\hat{Z}([0, \tau+1[)) .
$$

Next we note that

(1) One has $\hat{Z}([0, \tau+1[)=\hat{Z}([0, \tau])$.

(2) The set $\hat{Z}\left(\left[0, \tau+1[)\right.\right.$ is $f_{\varepsilon}$-invariant.

Item (11) is a consequence of

$$
\begin{aligned}
\hat{Z}([0, \tau+1[) & =\hat{Z}([0, \tau]) \cup \hat{Z}([\tau, \tau+1[) \\
& =\hat{Z}([0, \tau]) \cup \hat{Z}([0,1[) \quad(\text { Lemma } 7.7) \\
& =\hat{Z}([0, \tau]) \quad(1 \leqslant \tau) .
\end{aligned}
$$


Item (2) follows from Item (11) and

$$
\begin{aligned}
f_{\varepsilon}(\hat{Z}([0, \tau+1[)) & =f_{\varepsilon}(\hat{Z}([0, \tau])) \\
& =\hat{Z}([1, \tau+1]) \\
& =\hat{Z}([1, \tau]) \cup \hat{Z}([\tau, \tau+1]) \\
& =\hat{Z}([1, \tau]) \cup \hat{Z}([0,1]) \quad \text { (Lemma } 7.7) \\
& =\hat{Z}([0, \tau]) \quad(1 \leqslant \tau) \\
& =\hat{Z}([0, \tau+1[) .
\end{aligned}
$$

Item (2) and (7.64) yield

$$
\hat{\Gamma}=\hat{Z}([0, \tau+1[) .
$$

This last identity shows that $\hat{\Gamma}$ is a connected, compact ( $c f$. Item (1) subset of $\mathbb{R}^{2}$ which is $f_{\varepsilon}$-invariant.

Let us prove that $\hat{\Gamma}$ is a 1 -dimensional submanifold of $\mathbb{R}^{2}$. Since $\hat{Z}(\tau)=$ $\hat{Z}(0)$ (Lemma 7.5) one has

$$
\hat{Z}([0, \tau+1[)=\hat{Z}(] 0, \tau+1[)=\hat{Z}(] 0, \tau[) \cup \hat{Z}(] \tau-1, \tau+1[) .
$$

From Lemmata [7.2, 7.3 the set $\hat{Z}(] 0, \tau[)$ is a 1-dimensional submanifold of $\mathbb{R}^{2}$ as well as the set $\hat{Z}(] \tau-1, \tau+1[$ ) (note that $\hat{Z}(] \tau-1, \tau+1[$ ) $=$ $f_{\varepsilon}(\hat{Z}([\tau-2, \tau[))$. The intersection of these two sets is $\hat{Z}(] \tau, \tau+1[)$ and from Lemma 7.7 it is equal to $\hat{Z}(] 0,1[)$ which is a 1-dimensional submanifold of $\mathbb{R}^{2}$. As a consequence the union $\hat{Z}(] 0, \tau[) \cup \hat{Z}(] \tau-1, \tau+1[)$ is 1-dimensional submanifold of $\mathbb{R}^{2}$.

This concludes the proof of Proposition 7.1

\section{Proof of Theorem 2.1 (hence of Theorem @)}

As we have mentioned in Section 2.4 Theorem $\mathrm{A}$ follows from Theorem 2.1. we describe the proof of this latter result in this Section.

Let $r=k-k_{0}-2,|\varepsilon| \leqslant \varepsilon_{1}$ and $\nu \leqslant \nu_{1}$. Theorem 6.2 yields a set $\overline{\mathcal{G}}_{\varepsilon, \nu}$ of $C^{r}, \bar{f}_{\varepsilon}$-invariant graphs contained in $\left.(\mathbb{R} / \mathbb{Z}) \times\right] e^{-1} \nu, \nu[$, the union of which covers a set of positive Lebesgue measure.

In the previous section ( $c f$. Proposition 7.1), for all $\nu \in] 0, \nu_{1}[$, we have associated to each $\bar{f}_{\varepsilon}$-invariant graph $\bar{\gamma} \in \overline{\mathcal{G}}_{\varepsilon, \nu}$ an $f_{\varepsilon}$-invariant $C^{r}$-curve

$$
\hat{\Gamma}=\bigcup_{n \in \mathbb{Z}} f_{\varepsilon}^{n}(\hat{\gamma}), \quad \text { where } \quad \hat{\gamma}=h_{\varepsilon}^{-1}(\bar{\gamma}) .
$$

We denote by $\hat{\mathcal{G}}_{\varepsilon, \nu}$ the set of all such curves $\hat{\Gamma}$.

To prove Theorem [2.1] we just have to prove that for all $\nu \in] 0, \nu_{1}[$ 


$$
\text { (Positive measure) } \quad \operatorname{Leb}_{\mathbb{R}^{2}}\left(\bigcup_{\hat{\Gamma} \in \hat{\mathcal{G}}_{\varepsilon, \nu}} \hat{\Gamma}\right)>0
$$

and

$$
\text { (Accumulation) } \quad \lim _{\nu \rightarrow 0} \sup _{\hat{\Gamma} \in \hat{\mathcal{G}}_{\varepsilon, \nu}} \operatorname{dist}\left(\hat{\Gamma}, \Sigma_{\varepsilon}\right)=0 .
$$

8.1. Proof of (8.66) (Positive measure). This is a consequence of the inclusion $(c f .(8.65))$

$$
h_{\varepsilon}^{-1}\left(\bigcup_{\bar{\gamma} \in \overline{\mathcal{G}}_{\varepsilon, \nu}} \bar{\gamma}\right) \subset \bigcup_{\hat{\Gamma} \in \hat{\mathcal{G}}_{\varepsilon, \nu}} \hat{\Gamma}
$$

and of the fact that $\operatorname{Leb}_{2}\left(\bigcup_{\bar{\gamma} \in \overline{\mathcal{G}}_{\varepsilon, \nu}} \bar{\gamma}\right)>0$ (this is the content of Theorem 6.2).

8.2. Proof of (8.67) (Accumulation). Let $\left.\bar{\gamma} \in \overline{\mathcal{G}}_{\varepsilon, \nu}, \bar{\gamma} \subset(\mathbb{R} / \mathbb{Z}) \times\right] 0, \nu[$. From the definition (5.45) of the diffeomorphism $h_{\varepsilon}$ we see that for some positive constant $C_{\lambda}$ depending on $\lambda$ (cf. condition (H3)

$$
\hat{\gamma}=h_{\varepsilon}^{-1}(\bar{\gamma}) \subset\left\{(x, y) \in \tilde{\mathcal{F}}_{\varepsilon, y_{*}}, x y \in\right] 0, C_{\lambda} \nu[\} .
$$

On the other hand

$$
\hat{\Gamma}=\bigcup_{n \in \mathbb{Z}} f_{\varepsilon}^{n}(\hat{\gamma})=\left(\bigcup_{n \in \mathbb{Z}} f_{\varepsilon}^{n}(\hat{\gamma}) \cap V\right) \cup \bigcup_{\substack{n \in \mathbb{Z} \\ f_{\varepsilon}^{n}(\hat{\gamma}) \notin V}} f_{\varepsilon}^{n}(\hat{\gamma})
$$

From condition $(H 3)$ one has

$$
\bigcup_{n \in \mathbb{Z}} f_{\varepsilon}^{n}(\hat{\gamma}) \cap V \subset V \cap\{(x, y), x y \in] 0, C_{\lambda} \nu[\}
$$

hence, using Remark 2.2

$$
\operatorname{dist}\left(\bigcup_{n \in \mathbb{Z}} f_{\varepsilon}^{n}(\hat{\gamma}) \cap V, \Sigma_{\varepsilon} \cap V\right)=o_{\nu}(1) \text { (uniform in } \hat{\gamma} \text { ). }
$$

Now, recalling the definition (4.29) of the integer $N$ of Section 4.2 one has

$$
\bigcup_{\substack{n \in \mathbb{Z} \\ f_{\varepsilon}^{n}(\hat{\gamma}) \notin V}} f_{\varepsilon}^{n}(\hat{\gamma}) \subset \bigcup_{n=1}^{N} f_{\varepsilon}^{-n}(\hat{\gamma})
$$

and using the fact that $\operatorname{dist}\left(\hat{\gamma}, \Sigma_{\varepsilon} \cap\left[\left(x_{*}, 0\right), f_{\varepsilon}\left(x_{*}, 0\right)[)=o_{\nu}(1)\right.\right.$ one can see that ( $N$ is fixed)

$$
\operatorname{dist}\left(\bigcup_{\substack{n \in \mathbb{Z} \\ f_{\varepsilon}^{n}(\hat{\gamma}) \notin V}} f_{\varepsilon}^{n}(\hat{\gamma}), \Sigma_{\varepsilon} \cap \bigcup_{j=1}^{N} f_{\varepsilon}^{-1}\left(\left[\left(x_{*}, 0\right), f_{\varepsilon}\left(x_{*}, 0\right)[)\right)\right)=o_{\nu}(1)\right.
$$

where the previous limit is uniform in $\hat{\gamma}$. 
Equations (8.68), (8.69) give

$$
\operatorname{dist}\left(\hat{\Gamma}, \Sigma_{\varepsilon}\right)=o_{\nu}(1) .
$$

8.3. KAM circles for $f_{\varepsilon}$. Let $\hat{\Gamma}$ be a $C^{r}$ invariant curve for $f_{\varepsilon}$ of the form (8.65) and $g_{\hat{\Gamma}}$ the restriction of $f_{\varepsilon}$ to $\hat{\Gamma}$. The map $g_{\hat{\Gamma}}$ can be identified with a circle diffeomorphism. Similarly, the restriction of $\bar{f}_{\varepsilon}$ to the invariant curve $\bar{\gamma}$ yields a circle diffeomorphism $g_{\bar{\gamma}}$.

Let $\hat{\alpha}$ and $\bar{\alpha}$ are the rotation numbers of $g_{\hat{\Gamma}}$ and $g_{\bar{\gamma}}$.

Lemma 8.1. One has $\{1 / \hat{\alpha}\}=\bar{\alpha}$ (here $\{\cdot\}$ denotes the fractional part).

Proof. We refer to the renormalization procedure defined in Sections 4 and 5. Let $\hat{J}$ be the arc $\tilde{\mathcal{F}}_{\varepsilon, y_{*}} \cap \hat{\Gamma}$. The restriction on $\hat{J}$ of $\hat{f}_{\varepsilon}$, the first return map of $f_{\varepsilon}$ in $\tilde{\mathcal{F}}_{\varepsilon, y_{*}}$, defines a $C^{r}$ diffeomorphism of the abstract circle $\hat{J} / f_{\varepsilon}$. Classical arguments show that the rotation number of this circle diffeomorphism is equal to $\{1 / \hat{\alpha}\}$. On the other hand, after normalization of $f_{\varepsilon}$ by $h_{\varepsilon}(c f$. formula (5.46) $), \hat{\Gamma}$ is transported to $\bar{\gamma}$ and the $C^{r}$ diffeomorphism $\hat{f}_{\varepsilon}: \hat{J} / f_{\varepsilon} \rightarrow \hat{J} / f_{\varepsilon}$ to the circle diffeomorphism $\bar{f}_{\varepsilon}: \bar{J} / T_{1} \rightarrow \bar{J} / T_{1}$ where $\bar{J}=h_{\varepsilon}(\hat{J}) \subset \bar{\gamma}$ is a fundamental domain of $\bar{f} \mid \bar{\gamma}$. The rotation numbers of $\hat{f}_{\varepsilon}: \hat{J} / f_{\varepsilon} \rightarrow \hat{J} / f_{\varepsilon}$ and $\bar{f}_{\varepsilon}: \bar{J} / T_{1} \rightarrow \bar{J} / T_{1}$ are hence equal. But the rotation number of $\bar{f}_{\varepsilon}: \bar{J} / T_{1} \rightarrow \bar{J} / T_{1}$ is (same argument as before) equal to $\{1 / \bar{\alpha}\}$.

Since $\bar{\alpha}$ can be chosen in a fixed Diophantine class $D C(\kappa, \tau)$ (see Remark 6.1), the rotation number $\hat{\alpha}$ is Diophantine with the same exponent $\tau$. By Herman-Yoccoz Theorem on linearization of $C^{r}$-circle diffeomorphisms ([9], [22]), this implies that if $r$ is large enough (depending on $\tau$ which is fixed), the diffeomorphism $g_{\hat{\Gamma}}$ is linearizable; in other words, $\hat{\Gamma}$ is a $K A M$ curve. On the other hand one has a priori no control on the Diophantine constant of $\hat{\alpha}$.

This concludes the proof of Theorem 2.1 whence of Theorem A.

\section{Proof of Theorem B}

We construct in Subsection 9.1 a symplectic diffeomorphism $f_{\text {pert }}$ admitting a separatrix $\Sigma$ (see Figure 5) and depending on a ("large") parameter $M$. We renormalize $f_{\text {pert }}$ like in Sections 4 and 5] to get a diffeomorphism $\bar{f}_{\text {pert }}$ of an open annulus $\left.\mathbb{R} / \mathbb{Z} \times\right] 0, c[$. We prove in Proposition 9.3 of Subsection 9.2 that this renormalized diffeomorphism $\bar{f}_{\text {pert }}$ sends some graphs projecting on a fixed interval $J_{M}$ (see (9.81) on graphs which project on the whole circle and which are below the initial graphs we have started from, see Figure 6. We then iterate this procedure in Subsection 9.3 to find an orbit of $\bar{f}_{\text {pert }}$ accumulating the boundary $\mathbb{R} / \mathbb{Z} \times\{0\}$ of the aforementioned annulus: this prevents the existence of $\bar{f}_{\text {pert }}$-invariant curves close to this boundary and therefore of $f_{\text {pert }}$-invariant curves close to the separatrix $\Sigma$. The diffeomorphism $f_{\text {pert }}$ is the searched for example of Theorem $\mathrm{B}$. 
9.1. Construction of the example. We start with a smooth autonomous symplectic vector field of the form $X_{0}=J \nabla H_{0}$ where $H_{0}: \mathbb{R}^{2} \rightarrow \mathbb{R}$ satisfies on some neighborhood $V$ of $o=(0,0)$

$$
H_{0}(x, y)=x y \quad \text { on } V
$$

and has the property that $\Sigma=H_{0}^{-1}\left(H_{0}(0,0)\right)$ is compact and connected. The set $\Sigma$ is a separatrix of

$$
f_{\text {defin. }}^{=} \phi_{J \nabla H_{0}}^{1}
$$

associated to the hyperbolic fixed point $o$.

Fixing $x_{*}>0$ small enough we can define like in Section 4, for $y_{*}>0$ small enough, a fundamental domain $\tilde{\mathcal{F}}_{y_{*}}=\mathcal{F}_{y_{*}} \cup L_{y_{*}} \subset V$ where $\mathcal{F}_{y_{*}}$ is defined by $(a)-(d)$ (sec. 4.1) with $\phi_{J \nabla H_{0}}^{1}$ in place of $f_{\varepsilon}$. We can even assume that $\phi_{J \nabla H_{0}}^{-j}\left(\tilde{\mathcal{F}}_{y_{*}}\right) \subset V, j=1,2$. There exists $c_{*}>0$ such that the first return map

$$
\hat{f}: \tilde{\mathcal{F}}_{c * y *} \rightarrow \mathcal{F}_{y *}
$$

is well defined. We can renormalize $f=\phi_{J \nabla H_{0}}^{1}$ like in Section 5 by first normalizing $f(c f$. Lemma 5.3):

$$
h \circ f \circ h^{-1}=T_{1}
$$

where

$$
h: \mathcal{F}_{y_{*}} \rightarrow[0,1[\times] 0, c[
$$

is symplectic 12 and then setting (cf. (5.46) $)$

$$
\left.\bar{f} \underset{\text { defin. }}{=} h \circ \hat{f} \circ h^{-1}: \mathbb{R} / \mathbb{Z} \times\right] 0, \delta[\rightarrow \mathbb{R} / \mathbb{Z} \times] 0, c[.
$$

By (5.48) of Proposition 5.4 we have

$$
\bar{f}=T_{l}, \quad l(y)=\sigma(y)-\ln y
$$

for some smooth function $\sigma$.

We can assume that $h\left(\tilde{\mathcal{F}}_{y *}\right)=\left[0,1[\times] 0, c\left[\right.\right.$ and that $T^{-j}([0,1[\times] 0, c[) \subset$ $h^{-1}(V), j=1,2$.

We now construct a symplectic perturbation $f_{\text {pert }}: \mathbb{R}^{2} \rightarrow \mathbb{R}^{2}$ of $f$ which admits $\Sigma$ as a separatrix. We shall need first the following lemma.

Lemma 9.1. There exist $b \in(0,1)$ and a nonempty compact interval $I \subset$ ]0, 1 [ such that for any $M>0$, there exists a smooth function $\varphi_{M}: \mathbb{R} \rightarrow \mathbb{R}$ satisfying

(1) $\left.\varphi_{M}\right|_{I} \leqslant-b M$

(2) $\frac{b^{-1} M}{|I|} \geqslant-\left.\varphi_{M}^{\prime}\right|_{I} \geqslant \frac{M}{|I|}$

\footnotetext{
${ }^{12}$ See (5.45), (5.41) and the fact that we choose $q(s)=s$.
} 
(3) the map $s_{M}: \mathbb{R} \rightarrow \mathbb{R}$ defined by

$$
s_{M}(t)=\int_{0}^{t} e^{\varphi_{M}(u)} d u
$$

is an increasing smooth diffeomorphism of $\mathbb{R}$ that coincides with the identity on $\mathbb{R} \backslash[0,1]$.

Proof. See the Appendix C.

Let $\chi: \mathbb{R} \rightarrow \mathbb{R}$ be a smooth function equal to 1 on $[-c / 2, c / 2]$ and to 0 on $\mathbb{R} \backslash[-(3 / 4) c,(3 / 4) c]$ and define

$$
S_{M}(x, y)=\left(s_{M}(x) y\right) \chi(y)+x y(1-\chi(y)) .
$$

The canonical (hence symplectic) mapping $g_{M}$ associated to $S_{M}$ :

$$
g_{M}(x, y)=(\tilde{x}, \tilde{y}) \Longleftrightarrow\left\{\begin{array}{l}
x=\frac{\partial S_{M}}{\partial y}(\tilde{x}, y) \\
\tilde{y}=\frac{\partial S_{M}}{\partial \tilde{x}}(\tilde{x}, y)
\end{array}\right.
$$

is equal to the identity on $(\mathbb{R} \backslash[0,1]) \times[-c, c]$ and satisfies for $(x, y) \in$ $[0,1[\times] 0, c / 2[$

$$
\left\{\begin{array}{l}
\tilde{x}=s_{M}^{-1}(x) \\
\tilde{y}=s_{M}^{\prime} \circ s_{M}^{-1}(x) y .
\end{array}\right.
$$

The following symplectic perturbation of $f$

$$
\begin{gathered}
f_{\text {pert }}: \underset{\text { defin. }}{=} h^{-1} \circ\left(g_{M} \circ T_{1}\right) \circ h \\
\quad=\left(h^{-1} \circ g_{M} \circ h\right) \circ f
\end{gathered}
$$

(recall $h$ satisfies $(9.70)$ ) is thus defined on $\mathbb{R}^{2}$ and coincides with $f$ outside $f^{-1}\left(\tilde{\mathcal{F}}_{y_{*}}\right)$. Moreover, since $g_{M}(\mathbb{R} \times\{0\})=\mathbb{R} \times\{0\}$

$$
\Sigma \text { is a separatrix for } f_{\text {pert }} \text {. }
$$

Now, since $\tilde{\mathcal{F}}_{y_{*}}$ is a fundamental domain for $f_{\text {pert }}\left(f_{\text {pert }}\right.$ coincide with $f$ on $\left.\tilde{\mathcal{F}}_{y_{*}}\right)$, for some $c_{\text {pert }}>0$ small enough, the first return map

$$
\hat{f}_{\text {pert }}: f_{\text {pert }}^{-1}\left(\tilde{\mathcal{F}}_{c_{\text {pert }} y_{*}}\right) \rightarrow f_{\text {pert }}^{-1}\left(\tilde{\mathcal{F}}_{y_{*}}\right),
$$

is well defined and satisfies

$$
\hat{f}_{\text {pert }}=\left(\hat{f} \circ f^{-1}\right) \circ f_{\text {pert }} .
$$

In particular, on

one has (cf. (9.72), (9.73))

$$
[-1,0[\times] 0, c / 2[
$$

$$
\begin{aligned}
\bar{f}_{\text {pert }} \underset{\text { defin. }}{=} h \circ \hat{f}_{\text {pert }} \circ h^{-1} & =\bar{f} \circ T_{-1} \circ g_{M} \circ T_{1} \\
& =T_{l-1} \circ g_{M} \circ T_{1}
\end{aligned}
$$

and

$$
\left.\bar{f}_{\text {pert }}: \mathbb{R} \times\right] 0, c / 2[\rightarrow \mathbb{R} \times] 0, c\left[\text { satisfies } \quad \bar{f}_{\text {pert }} \circ T_{1}=T_{1} \circ \bar{f}_{\text {pert }} ;\right.
$$




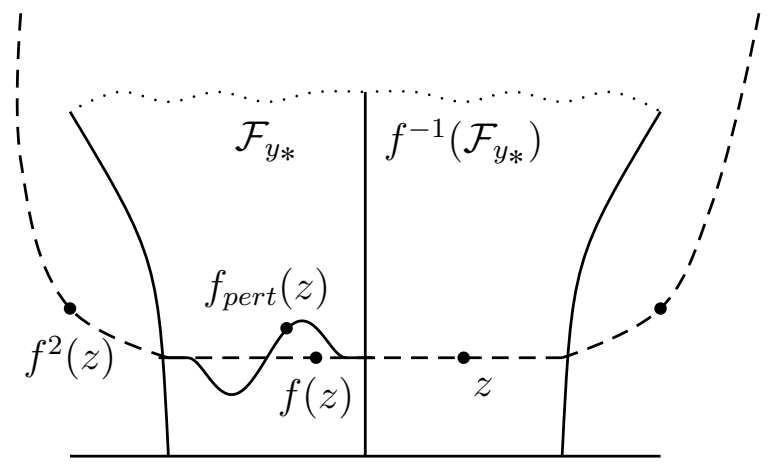

FigurE 5. The perturbed map $f_{\text {pert }}$.

in particular, it defines a smooth map $(\mathbb{R} / \mathbb{Z}) \times] 0, c / 2[\rightarrow(\mathbb{R} / \mathbb{Z}) \times] 0, c[$.

Note that since $g_{M}$ is the identity outside $[0,1] \times[-c, c]$, it admits a $T_{1}$ periodization $\tilde{g}_{M}: \mathbb{R} \times[-c, c] \rightarrow \mathbb{R} \times[-c, c]$ (which means that $\tilde{g}_{M}$ and $g_{M}$ coincide on $[0,1] \times[-c, c]$ and $\tilde{g}_{M}$ commutes with $\left.T_{1}\right)$. This $\tilde{g}_{M}$ is defined by the same formula (9.75) as $g_{M}$ where now the new function $\tilde{s}_{M}$ involved in (9.74) is the $\mathbb{Z}$-periodization of $s_{M}$. To simplify the notation we shall continue to denote $\tilde{g}_{M}$ and $\tilde{s}_{M}$ by $g_{M}$ and $s_{M}$.

Let

$$
t:=t(x):=s_{M}^{-1}(x+1)
$$

Lemma 9.2. For $(x, y) \in\left[-1,0[\times] 0, c / 2\left[\right.\right.$, the point $(\bar{x}, \bar{y}):=\bar{f}_{\text {pert }}(x, y)$ satisfies with the notation 9.79)

$$
\left\{\begin{array}{l}
\bar{x}=t-1+\sigma\left(s_{M}^{\prime}(t) \times y\right)-\ln \left(s_{M}^{\prime}(t)\right)-\ln y \\
\ln \bar{y}=\ln \left(s_{M}^{\prime}(t)\right)+\ln y
\end{array}\right.
$$

Proof. Let $(x, y) \in\left[-1,0[\times] 0, c / 2\left[\right.\right.$; with the notations $\left(x_{1}, y_{1}\right)=\left(g_{M} \circ\right.$ $\left.T_{1}\right)(x, y)=g_{M}(x+1, y)$, one has from (9.78) $(\bar{x}, \bar{y})=T_{l-1}\left(x_{1}, y_{1}\right)$ and from (9.76), (9.73)

$$
\left\{\begin{array} { l } 
{ x _ { 1 } = s _ { M } ^ { - 1 } ( x + 1 ) } \\
{ y _ { 1 } = s _ { M } ^ { \prime } \circ s _ { M } ^ { - 1 } ( x + 1 ) \times y }
\end{array} \text { and } \quad \left\{\begin{array}{l}
\bar{x}=x_{1}-1+\sigma\left(y_{1}\right)-\ln y_{1} \\
\bar{y}=y_{1} .
\end{array}\right.\right.
$$

hence (9.80).

9.2. Image of a piece of graph by $\bar{f}_{\text {pert }}$. We take $M>0$ (from Lemma 9.1) large enough and we define

$$
J_{M}=s_{M}(I)-1 \subset[-1,0[
$$


where $I$ is the interval introduced in Lemma 9.1.

If $y: J \rightarrow \mathbb{R}_{+}^{*}, x \rightarrow y(x)$ is a differentiable function, we denote by $\gamma_{J, y}$ its graph:

$$
\gamma_{J, y}=\{(x, y(x)), x \in J\} \subset[-1,0[\times] 0, c / 2[\text {. }
$$

Proposition 9.3. There exists a constant $y_{\text {pert }}>0$ for which the following holds. Assume that $\left.y: J_{M} \rightarrow\right] 0, y_{\text {pert }}[, x \mapsto y(x)$ is a differentiable function such that

$$
\forall x \in J_{M}, \quad\left|\frac{d \ln y}{d x}+1\right| \leqslant 1 / 2 .
$$

Then, $\bar{f}_{\text {pert }}\left(\gamma_{J_{M}, y}\right)+(\mathbb{Z}, 0)$ contains the graph $\gamma_{[-1,0[, \bar{y}}$ of a differentiable function $\bar{y}:\left[-1,0\left[\rightarrow \mathbb{R}_{+}^{*}\right.\right.$ (see Figure [6)

$$
\gamma_{[-1,0[, \bar{y}}=\{(\bar{x}, \bar{y}(\bar{x})), \bar{x} \in[-1,0[\},
$$

such that

$$
\begin{aligned}
& \forall \bar{x} \in\left[-1,0\left[, \quad\left|\frac{d \ln \bar{y}}{d \bar{x}}+1\right| \leqslant 1 / 2 ;\right.\right. \\
& \sup _{\bar{x} \in[-1,0[} \ln \bar{y}(\bar{x}) \leqslant \sup _{x \in J_{M}} \ln y(x)-b M .
\end{aligned}
$$

Moreover, for some interval $J_{M}^{1} \subset J_{M}$ one has

$$
\gamma_{[-1,0[, \bar{y}}=\bar{f}_{\text {pert }}\left(\gamma_{J_{1}, y}\right) \text {. }
$$

We prove this Proposition in subsection 9.2.2,

9.2.1. Preliminary results. If we introduce the variable

$$
\varphi=\ln \left(s_{M}^{\prime}(t)\right)=\ln s_{M}^{\prime} \circ s_{M}^{-1}(x+1) \quad\left(\text { recall } t=s_{M}^{-1}(x+1)\right)
$$

we can write (9.80) as

$$
(\bar{x}, \bar{y})=f_{\text {pert }}(x, y(x)) \Longleftrightarrow\left\{\begin{array}{l}
\bar{x}=t-1+\sigma\left(e^{\varphi} \times y(x)\right)-\varphi-\ln y(x) \\
\ln \bar{y}=\varphi+\ln y(x) .
\end{array}\right.
$$

Note that the maps $I \ni t \mapsto \varphi=\ln s_{M}^{\prime}(t) \in \varphi(I)$ and $J_{M} \ni x \mapsto \varphi=$ $\ln s_{M}^{\prime} \circ s_{M}^{-1}(x+1) \in \varphi_{M}(I)$ are smooth diffeomorphisms. In particular, the maps $\varphi_{M}(I) \ni \varphi \mapsto \bar{x}$ and $\varphi_{M}(I) \ni \varphi \mapsto \ln y, \varphi_{M}(I) \ni \varphi \mapsto \ln \bar{y}$ are well defined and smooth.

Lemma 9.4. For any $\varphi$ such that $t \in I$ one has

$$
\left|\frac{d t}{d \varphi}\right| \leqslant|I| / M \leqslant 1 / 4
$$

Proof. This follows from the identity (recall $\varphi=\ln \left(s_{M}^{\prime}(t)\right), s_{M}^{\prime}=e^{\varphi_{M}}$ )

$$
\frac{d t}{d \varphi}=\frac{1}{d \varphi / d t}=\frac{1}{\varphi_{M}^{\prime}(t)}
$$


and the estimates given by the second item of Lemma 9.1 ( $M$ is assumed to be large enough).

Lemma 9.5. One has

$$
\begin{aligned}
& \sup _{\varphi_{M}(I)}\left|\frac{d \bar{x}}{d \varphi}+1\right| \leqslant 1 / 4, \\
& \sup _{\varphi_{M}(I)}\left|\frac{d \ln \bar{y}}{d \varphi}-1\right| \leqslant 1 / 4 .
\end{aligned}
$$

Proof. Indeed, from (9.85)

$$
\begin{aligned}
\frac{d \bar{x}}{d \varphi} & =\frac{d t}{d \varphi}+e^{\varphi} \sigma^{\prime}\left(e^{\varphi} \times y\right) \frac{d y}{d \varphi}-1-\frac{d \ln y}{d \varphi} \\
& =\frac{d t}{d \varphi}+y e^{\varphi} \sigma^{\prime}\left(e^{\varphi} \times y\right) \frac{d \ln y}{d \varphi}-1-\frac{d \ln y}{d \varphi} \\
& =-1+A
\end{aligned}
$$

with

$$
A=\frac{d t}{d \varphi}+y e^{\varphi} \sigma^{\prime}\left(e^{\varphi} \times y\right) \frac{d \ln y}{d \varphi}-\frac{d \ln y}{d \varphi}
$$

Note that (recall $x=s_{M}(t)-1, s_{M}^{\prime}=e^{\varphi_{M}}$ )

$$
\frac{d \ln y}{d \varphi}=\frac{d \ln y}{d x} \frac{d x}{d t} \frac{d t}{d \varphi}=\frac{d \ln y}{d x} e^{\varphi} \frac{d t}{d \varphi}
$$

so, by Lemma 9.4

$$
|A| \leqslant(|I| / M)+\left(y e^{-b M}\left\|\sigma^{\prime}\right\|_{0}+1\right) e^{-b M}(|I| / M)\left|\frac{d \ln y}{d x}\right|
$$

and if $M$ is large enough

$$
|A| \leqslant 1 / 4
$$

In a similar way

$$
\frac{d \ln \bar{y}}{d \varphi}=1+\frac{d \ln y}{d x} \frac{d x}{d t} \frac{d t}{d \varphi}=1+\frac{d \ln y}{d x} e^{\varphi} \frac{d t}{d \varphi}=1+B
$$

with

$$
|B| \leqslant 2 e^{-b M} \times(1 / 4) \leqslant 1 / 4 \quad(M \gg 1) .
$$

9.2.2. Proof of Proposition 9.3. From (9.86) of Lemma 9.5 we see that the $\operatorname{map} \varphi_{M}(I) \ni \varphi \mapsto \bar{x} \in \mathbb{R}$ is a diffeomorphism onto its image $\bar{J}_{M} \subset \mathbb{R}$, hence the maps $J_{M} \ni x \mapsto \bar{x} \in \bar{J}_{M}$ and $I \ni t \mapsto \bar{x} \in \bar{J}_{M}$ are diffeomorphisms. Note that from (9.86) one has

$$
\left|\bar{J}_{M}\right| \geqslant(3 / 4)\left|\varphi_{M}(I)\right|
$$

and from item (2) of Lemma 9.1 one has

$$
\left|\bar{J}_{M}\right| \geqslant(3 / 4)(M /|I|) \times|I|>2 ;
$$




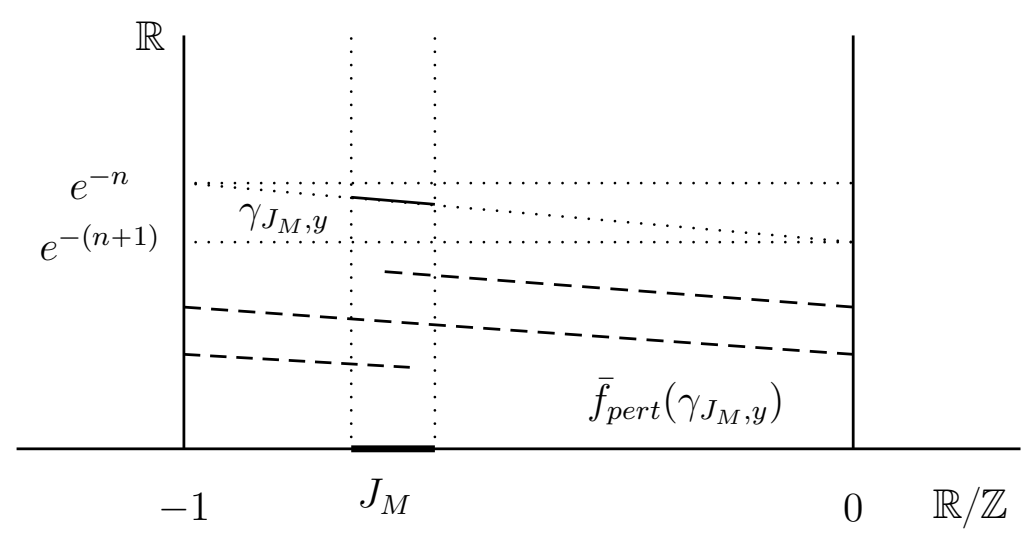

Figure 6 . The image of the graph $\gamma_{J_{M}, y}$ by the diffeomorphism $\bar{f}_{\text {pert }}$.

there thus exists an interval $J_{M}^{1} \subset J_{M}$ such that the map $J_{M}^{1} \ni x \mapsto \bar{x} \in$ $n+[-1,0[$ (for some $n \in \mathbb{Z}$ ) is a differentiable homeomorphism. Replacing $\bar{y}(\bar{x})$ by $\bar{y}(\bar{x}+n)$ shows (9.84).

We now prove (9.82): for $\bar{x} \in[-1,0[$

$$
\left|\frac{d \ln \bar{y}}{d \bar{x}}+1\right| \leqslant 1 / 2 \text {. }
$$

Indeed, let $I_{1} \subset I$ be the image of $\left[0,1\left[\right.\right.$ by $\bar{J}_{M} \ni \bar{x} \mapsto t \in I$; from Lemma 9.5. for any $\varphi \in \varphi_{M}\left(I_{1}\right)$ one has for some $A, B \in[0,1 / 4]$

$$
\frac{d \bar{x}}{d \varphi}=-1+A, \quad \frac{d \ln \bar{y}}{d \varphi}=1+B
$$

so that

$$
\left|\frac{d \ln \bar{y}}{d \bar{x}}+1\right|=\left|\left(\frac{d \ln \bar{y}}{d \varphi} / \frac{d \bar{x}}{d \varphi}\right)+1\right|=\left|\frac{1+B}{-1+A}+1\right| \leqslant 1 / 2 .
$$

The preceding discussion shows that the map $\bar{y}:[-1,0[\ni \bar{x} \mapsto \bar{y}(\bar{x})$ is a well defined differentiable function, that its graph is included in $f_{\text {pert }}\left(\gamma_{J_{M}, y}\right)+$ $(\mathbb{Z}, 0)$ and that $(9.82)$ holds.

There remains to prove (9.83). By the second equality of (9.85), if $(\bar{x}, \bar{y}(\bar{x}))=$ $f_{\text {pert }}(x, y)$ one has

$$
\ln \bar{y}(\bar{x}) \leqslant \ln y(x)-b M \leqslant \sup _{x \in J_{M}} \ln y-b M
$$

and as a consequence since the map $J_{M} \supset J_{M}^{1} \ni x \mapsto \bar{x} \in[-1,0[$ is a bijection, (9.83) holds.

9.3. End of the proof of Theorem $\mathbf{B}$. We shall prove that if $M$ is large enough, the diffeomorphism $f_{\text {pert }}$ constructed in Subsection 9.1 provides the searched for example of Theorem B. 
Let $M$ be large enough and $\left.y_{0} \in\right] 0, y_{\text {pert }}[$; we define the function

$$
y_{0}:\left[-1,0\left[\rightarrow \mathbb{R}, \quad x \mapsto y_{0} e^{-x} .\right.\right.
$$

Using inductively Proposition 9.3 we construct differentiable functions

$$
y_{n}:[-1,0[\rightarrow \mathbb{R}
$$

such that for every $n \in \mathbb{N}^{*}$

$$
\begin{aligned}
& \forall x \in J_{M}, \quad\left|\frac{d \ln y_{n}}{d x}+1\right| \leqslant 1 / 2 \\
& \gamma_{\left[-1,0\left[, y_{n}\right.\right.} \subset \bar{f}_{\text {pert }}\left(\gamma_{J_{M}, y_{n-1}}\right)+(\mathbb{Z}, 0) \\
& \sup _{x \in[-1,0[} \ln y_{n}(x) \leqslant \sup _{x \in J_{M}} \ln y_{n-1}(x)-b M .
\end{aligned}
$$

Inclusion (9.93) implies the existence of a decreasing sequence of nonempty compact intervals $K_{n} \subset J_{M}$ such that

$$
\gamma_{[-3 / 4,-1 / 4], y_{n}}=\bar{f}_{\text {pert }}^{n}\left(\gamma_{K_{n}, y_{0}}\right) \bmod (\mathbb{Z}, 0) .
$$

In particular if $x_{\infty} \subset \bigcap_{n \in \mathbb{N}^{*}} K_{n}$ one has

$$
\forall n \in \mathbb{N}^{*}, \quad \bar{f}_{\text {pert }}^{n}\left(\left(x_{\infty}, y_{0}\right)\right) \in \gamma_{[-3 / 4,-1 / 4], y_{n}} \subset \gamma_{\left[-1,0\left[, y_{n}\right.\right.} \bmod (\mathbb{Z}, 0) .
$$

From (9.94)

$$
\sup _{x \in[-1,0[} y_{n}(x) \leqslant e^{-n b M} y_{0}
$$

hence, using (9.95) we see that $\bar{f}_{\text {pert }}^{n}\left(\left(x_{\infty}, y_{0}\right)\right)$ accumulates $\mathbb{R} \times\{0\}$ :

$$
\bar{f}_{\text {pert }}^{n}\left(\left(x_{\infty}, y_{0}\right)\right) \in\left[-1,0[\times] 0, e^{-n b M} y_{0}[\bmod (\mathbb{Z}, 0) .\right.
$$

As a consequence of (9.77) and of the fact that for some constant $C>0$

$$
\forall \nu \in] 0, c\left[, \quad h^{-1}\left(\left[-1,0[\times] 0, \nu[) \subset f_{\text {pert }}^{-1}\left(\hat{\mathcal{F}}_{C \nu}\right),\right.\right.\right.
$$

(this is due to the fact that the diffeomorphism $h$ given by (9.71) is indeed defined on a neighborhood of $\tilde{\mathcal{F}}_{y_{*}}$ ) one has

$$
\hat{f}_{\text {pert }}^{n}\left(h^{-1}\left(x_{\infty}, y_{0}\right)\right) \in f_{\text {pert }}^{-1}\left(\hat{\mathcal{F}}_{C e^{-n b M} y_{0}}\right) .
$$

Since $\hat{f}_{\text {pert }}$ is the first return map of $f_{\text {pert }}$ in $f_{\text {pert }}^{-1}\left(\hat{\mathcal{F}}_{y_{*}}\right)$, there exists a sequence $\left(p_{n}\right)_{n \in \mathbb{N}} \in \mathbb{N}^{\mathbb{N}}, \lim _{n \rightarrow \infty} p_{n}=\infty$ such that

$$
f_{\text {pert }}^{p_{n}}\left(h^{-1}\left(x_{\infty}, y_{0}\right)\right) \in f_{\text {pert }}^{-1}\left(\hat{\mathcal{F}}_{C e^{-n b M} y_{0}}\right) .
$$

But this last fact prevents the existence of invariant circles in $\Delta_{\Sigma}$ accumulating the separatrix $\Sigma$ of $f_{\text {pert }}$. More precisely, let $W$ be a neighborhood of $\Sigma$ in $\Sigma \cup \Delta_{\Sigma}$ (we recall that $\Delta_{\Sigma}$ is the bounded connected component of $\left.\mathbb{R}^{2} \backslash \Sigma\right)$ such that

$$
h^{-1}\left(x_{\infty}, y_{0}\right) \notin W .
$$

We claim that $W \backslash \Sigma$ does not contain any $f_{\text {pert }}$-invariant circle $\Gamma$. Indeed, if this were not the case, the topological annulus $\mathcal{A} \subset W$ having $\Sigma$ and $\Gamma$ 


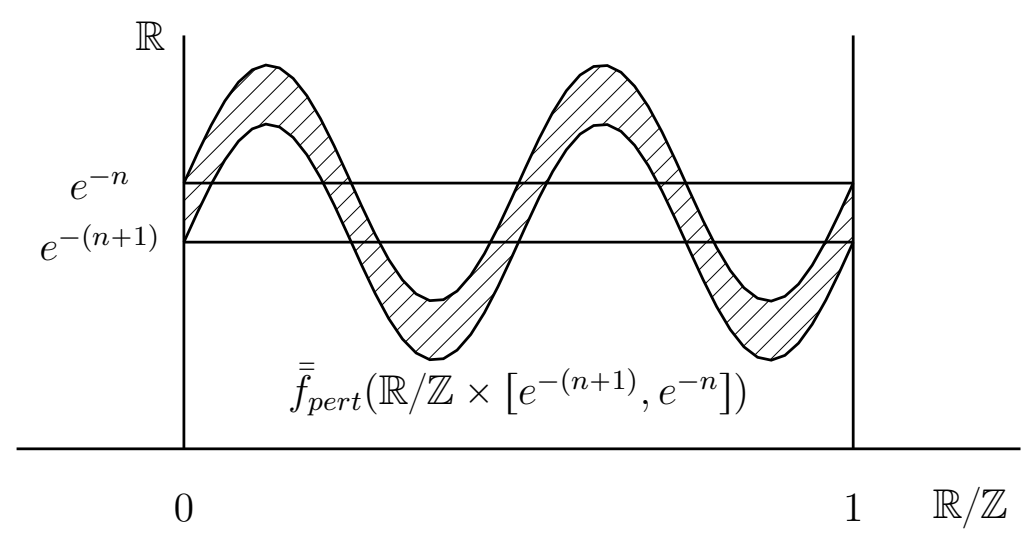

Figure 7. The diffeomorphism $\overline{\bar{f}}_{\text {pert }}$ on $\mathbb{R} / \mathbb{Z} \times\left[e^{-(n+1)}, e^{-n}\right]$. Compare with Figures [6] and 4 .

for boundaries would be $f_{\text {pert }}$-invariant (by topological degree theory). But this is impossible since one would have at the same time

$$
h^{-1}\left(x_{\infty}, y_{0}\right) \notin \mathcal{A} \quad \text { and } \quad f_{\text {pert }}^{p_{n}}\left(h^{-1}\left(x_{\infty}, y_{0}\right)\right) \in \mathcal{A}
$$

for some large $p_{n}$ (see (9.97).

Remark 9.1. If we define the renormalization $\overline{\bar{f}}_{\text {pert }}$ of $f_{\text {pert }}$ by considering the first return map of $f_{\text {pert }}$ in $\mathcal{F}_{y_{*}}$ instead of $f_{\text {pert }}^{-1}\left(\mathcal{F}_{y_{*}}\right)$ as we have done to construct $\bar{f}_{\text {pert }}$, the dynamics of $\overline{\bar{f}}_{\text {pert }}$ looks like more the one pictured in Figure 7. The comparison of this picture and the one of Figure 4 illustrates the effect of the perturbative assumption in Theorem A.

\section{Appendix A. Proof of Lemma 2.2}

We write for $j \geqslant 2$

$$
H_{\varepsilon}^{t}(z)=\lambda_{\varepsilon} z_{1} z_{2}+\sum_{2 \leqslant i \leqslant[j / 2]} a_{\varepsilon, i} \times\left(z_{1} z_{2}\right)^{i}+\sum_{\substack{i_{1}, i_{2} \in \mathbb{N} \\ i_{1}+i_{2}=j+1}} h_{\varepsilon, i_{1}, i_{2}}(t) z_{1}^{i_{1}} z_{2}^{i_{2}}+O^{j+2}(z)
$$

where $a_{\varepsilon, i} \in \mathbb{R}$ and the $h_{\varepsilon, i_{1}, i_{2}}(\cdot)$ are smooth 1-periodic functions. We define

$$
H_{\varepsilon, 2}(z)=\lambda_{\varepsilon} z_{1} z_{2} .
$$

We first observe that if $G_{\varepsilon}^{t}$ is a solution of

$$
\left\{\begin{array}{l}
G_{\varepsilon}^{t}(z)=O^{j+1}(z) \\
H_{\varepsilon}^{t}(z)+\partial_{t} G_{\varepsilon}^{t}(z)+\left\{G_{\varepsilon}^{t}, H_{\varepsilon, 2}^{t}\right\}(z)=\tilde{q}_{\varepsilon}\left(z_{1} z_{2}\right)
\end{array}\right.
$$


for some $\tilde{q}_{\varepsilon}(u)=\lambda_{\varepsilon} u+\sum_{2 \leqslant i \leqslant[(j+1)] / 2} \tilde{a}_{\varepsilon, i} \times u^{i}, \tilde{a}_{\varepsilon, i} \in \mathbb{R}$, then $G_{\varepsilon}^{t}$ solves (2.15). We then have to solve (A.98) for some $\tilde{q}_{\varepsilon}$ and some $G_{\varepsilon}^{t}$ of the form

$$
\begin{aligned}
& \tilde{q}_{\varepsilon}(u)=\lambda_{\varepsilon} u+\sum_{2 \leqslant i \leqslant[(j+1)] / 2} \tilde{a}_{\varepsilon, i} \times u^{i} \\
& G_{\varepsilon}^{t}(z)=\sum_{i_{1}+i_{2}=j+1} g_{\varepsilon, i_{1}, i_{2}}(t) z_{1}^{i_{1}} z_{2}^{i_{2}}=O^{j+1}(z)
\end{aligned}
$$

where the $g_{\varepsilon, i_{1}, i_{2}}(\cdot)$ are 1-periodic. This amounts to finding 1-periodic solutions to the equations

$$
\begin{array}{lr}
h_{\varepsilon, i_{1}, i_{2}}(t)+\partial_{t} g_{\varepsilon, i_{1}, i_{2}}(t)-\lambda_{\varepsilon}\left(i_{1}-i_{2}\right) g_{\varepsilon, i_{1}, i_{2}}(t)=0 & \text { if } i_{1} \neq i_{2} \\
h_{\varepsilon, i, i}(t)+\partial_{t} g_{\varepsilon, i, i}(t)=\tilde{a}_{\varepsilon, i} & \text { if } i_{1}=i_{2}=i
\end{array}
$$

for each couple $\left(i_{1}, i_{2}\right) \in \mathbb{N}^{2}$ such that $i_{1}+i_{2}=j+1$. Note that in (A.100) this last equality occurs only if $j+1$ is even and $i=(j+1) / 2$. Equation (A.100) is then easily solved by setting

$$
\tilde{a}_{\varepsilon, i}=\int_{\mathbb{R} / \mathbb{Z}} h_{\varepsilon, i, i}(t) d t, \quad g_{\varepsilon, i, i}(t)=-\int_{0}^{t}\left(h_{\varepsilon, i, i}(s)-\tilde{a}_{\varepsilon, i}\right) d s .
$$

Equations (A.99) always admit unique 1-periodic solutions of the form

$$
\left\{\begin{array}{l}
g_{\varepsilon, i_{1}, i_{2}}(t)=e^{\lambda_{\varepsilon}\left(i_{1}-i_{2}\right) t} c_{\varepsilon, i_{1}, i_{2}}-\int_{0}^{t} e^{(t-s) \lambda_{\varepsilon}\left(i_{1}-i_{2}\right)} h_{\varepsilon, i_{1}, i_{2}}(s) d s \\
\text { where } c_{\varepsilon, i_{1}, i_{2}}=\left(e^{\lambda_{\varepsilon}\left(i_{1}-i_{2}\right)}-1\right)^{-1} \int_{0}^{1} e^{(1-s) \lambda_{\varepsilon}\left(i_{1}-i_{2}\right)} h_{\varepsilon, i_{1}, i_{2}}(s) d s .
\end{array}\right.
$$

In the preceding solutions, the dependence on $\varepsilon$ is smooth and if, for $\varepsilon=0$, the functions $h_{0, i_{1}, i_{2}}$ do not depend on $t$ we see that $g_{0, t_{1}, t_{2}}$ is a constant.

This concludes the proof of Lemma 2.2.

\section{ApPendix B. Extension of SYMPLECTIC DIFFEOMORPHisms}

Lemma B.1. Let $\left(\Theta_{\varepsilon}\right)_{\varepsilon \in]-\varepsilon_{0}, \varepsilon_{0}[}$, be a smooth (or continuous) family of $C^{k}$ symplectic diffeomorphisms $C^{1}$-close to the identity, defined on some open disk $D(o, \delta)$ of $\mathbb{R}^{2}$ and such that $\Theta_{\varepsilon}(o)=o$. Then, there exists $\left(\tilde{\Theta}_{\varepsilon}\right)_{\varepsilon \in]-\varepsilon_{0}, \varepsilon_{0}[} a$ smooth (or continuous) family of $C^{k}$ symplectic diffeomorphisms of $\mathbb{R}^{2}$ such that on $D(o, \delta / 2)$ one has $\tilde{\Theta}_{\varepsilon}=\Theta_{\varepsilon}$.

Proof. We use the notation $\Theta_{\varepsilon}(x, y)=(\tilde{x}, \tilde{y})$. Since $\Theta_{\varepsilon}$ is symplectic the 1 -form $\tilde{y} d \tilde{x}-y d x$ is closed and defined on a disk $D(o, 4 \delta / 5)$ of center $o$ and radius $4 \delta / 5$ (we assume $\Theta_{\varepsilon} C^{1}$-close to the identity so that we can use the Implicit Function Theorem). It is hence locally exact and there exists a function $S_{\varepsilon}(y, \tilde{y})$ such that $\tilde{y} d \tilde{x}-y d x=d S_{\varepsilon}$. Now the function $F_{\varepsilon}(x, \tilde{y})=-S_{\varepsilon}(y, \tilde{y})+(\tilde{x}-x) \tilde{y}$ is defined on $D(0,3 \delta / 4)$ and satisifes $(y-$ $\tilde{y}) d x+(\tilde{x}-x) d \tilde{y}=d F_{\varepsilon}$ or equivalently

$$
\Theta_{\varepsilon}(x, y)=(\tilde{x}, \tilde{y}) \Longleftrightarrow\left\{\begin{array}{l}
\tilde{x}=x+\partial_{\tilde{y}} F_{\varepsilon}(x, \tilde{y}) \\
y=\tilde{y}+\partial_{x} F_{\varepsilon}(x, \tilde{y}) .
\end{array}\right.
$$


Note that we can choose choose $\left(F_{\varepsilon}\right)_{\varepsilon}$ as a $C^{k}$-family of $C^{k+1}$-functions such that $F_{\varepsilon}(o)=0, D F_{\varepsilon}(o)=0$.

We can then choose $\chi: \mathbb{R}^{2} \rightarrow \mathbb{R}$ a smooth function which is equal to 1 on $D(o, 2 \delta / 3)$ and 0 outside $D(o, 3 \delta / 4)$, set

$$
\tilde{F}_{\varepsilon}=\chi \times F_{\varepsilon}
$$

and define $\tilde{\Theta}_{\varepsilon}$ by $\left(\right.$ B.101) with $F_{\varepsilon}$ replaced by $\tilde{F}_{\varepsilon}$. The family of diffeomorphisms $\left(\tilde{\Theta}_{\varepsilon}\right)_{\varepsilon}$ is a smooth (or continuous) family of exact symplectic $C^{k}$-diffeomorphisms.

\section{Appendix C. Proof of Lemma 9.1}

Let $\chi: \mathbb{R} \rightarrow[0,1]$ be a smooth even function with support in $[-1 / 2,1 / 2]$ such that $\chi(0)=1$ and which is increasing on $[-1 / 2,0]$. There exists $\alpha \in$ ]0,1/4[ such that for all $x \in]-2 \alpha, 2 \alpha[$ one has $\chi(x)>1 / 2$ and

$$
\beta_{\min }:=\min _{[-2 \alpha,-\alpha]} \chi^{\prime}>0, \quad \beta_{\max }:=\max _{[-2 \alpha,-\alpha]} \chi^{\prime}>0
$$

We define for $\rho \in] 0,1 / 12]$ and $C_{M}>0$

$$
\varphi_{M}(x)=a\left(\rho, C_{M}\right) \chi\left(\frac{x-1 / 3}{1 / 12}\right)-C_{M} \chi\left(\frac{x-2 / 3}{\rho}\right),
$$

where $a\left(\rho, C_{M}\right)>0$ is chosen so that

$$
\int_{0}^{1} e^{\varphi_{M}(u)} d u=1
$$

Let $I=(2 / 3)+]-2 \alpha \rho,-\alpha \rho[$. For $x \in I$ one has

$$
\begin{aligned}
& \varphi_{M}(x) \leqslant-C_{M} / 2=-C_{M} \alpha \beta_{\text {min }} /\left(2 \alpha \beta_{\text {min }}\right) \\
& \varphi_{M}^{\prime}(x) \leqslant-\left(C_{M} / \rho\right) \beta_{\text {min }}=-\left(C_{M} \alpha \beta_{\text {min }}\right) /(\alpha \rho)=-C_{M} \alpha \beta_{\text {min }} /|I| \\
& \varphi_{M}^{\prime}(x) \geqslant-\left(C_{M} / \rho\right) \beta_{\max }=-\left(C_{M} \alpha \beta_{\max }\right) /(\alpha \rho)=-\left(\beta_{\max } / \beta_{\min }\right) C_{M} \alpha \beta_{\text {min }} /|I| .
\end{aligned}
$$

Fixing $\rho$ (for example $\rho=1 / 12$ ) and taking

$$
b^{-1}=\max \left(\frac{\beta_{\text {max }}}{\beta_{\text {min }}}, 2 \alpha \beta_{\text {min }}\right), \quad C_{M}=\frac{M}{\alpha \beta_{\text {min }}},
$$

provides the first two items of Lemma 9.1.

Let us check the third item is satisfied. From the definition of $s_{M}$ one has $s_{M}^{\prime}(x)=e^{\varphi_{M}(x)}=1$ for $x \notin[0,1]$. Since $s_{M}(0)=0$ one has $s_{M}(x)=x$ for $x \leqslant 0$. Similarly since

$$
s_{M}(1)=\int_{0}^{1} e^{\varphi_{M}(u)} d u=1
$$

we have $s_{M}(x)=x$ for $x \geqslant 1$.

Since in any case $s^{\prime}(x)>0$, this concludes the proof of Lemma 9.1. 


\section{REFERENCES}

[1] S. Angenent, A remark on the topological entropy and invariant circles of an area preserving twist map. Twist mappings and their applications, 1-5, IMA Vol. Math. Appl., 44, Springer, New York, 1992.

[2] M.-C. Arnaud, Three results on the regularity of the curves that are invariant by an exact symplectic twist map, Publ. Math. Inst. Hautes Etudes Sci. 109, (2009) $1-17$.

[3] M.-C. Arnaud, A non-differentiable essential irrational invariant curve for a $C^{1}$ symplectic twist map, Journal of modern Dynamics 5, no. 3 (2011) 583-591.

[4] M.-C. Arnaud, Boundaries of instability zones for symplectic twist maps, Journal of the Institute of Mathematics of Jussieu, 13 (2013), issue 01, 19-41.

[5] A. Avila, B. Fayad, Non-differentiable irrational curves for $C^{1}$ twist map, https://arxiv.org/abs/1909.13556.

[6] A. Avila, R. Krikorian, Reducibility or non-uniform hyperbolicity for quasi-periodic Schrödinger cocycles, Annals of Mathematics (2) 164, no. 3, 911-940, (2006).

[7] A. Banyaga, R. de la Llave, C. E. Wayne, Cohomology Equations Near Hyperbolic Points and Geometric Versions of Sternberg Linearization Theorem, Journ. Geom. Anal., 6, nº (1996), 613-649.

[8] M. Chaperon, Géométrie differentielle et singularités des systèmes dynamiques. Astérisque 138-139 (1986).

[9] M. Herman, Sur la conjugaison différentiable des difféomorphismes du cercle à des rotations. Inst. Hautes Études Sci. Publ. Math. No. 49 (1979), 5-233.

[10] M. R. Herman. Sur les courbes invariantes par les difféomorphismes de l'anneau. Vol. 1. With an appendix by Albert Fathi. Astérisque, 103-104. Société Mathématique de France, Paris, 1983. i+221 pp.

[11] M. R. Herman, Some open problems in dynamical systems. Proceedings of the International Congress of Mathematicians, Vol. II (Berlin, 1998). Doc. Math. 1998, Extra Vol. II, 797-808.

[12] B. Fayad, R. Krikorian, Herman's last geometric theorem, Annales de l'Ecole Normale Supérieure (4) 42, no 2, pp. 193-219 (2009)

[13] A. Katok, Lyapunov exponents, entropy and periodic orbits for diffeomorphisms. Inst. Hautes Etudes Sci. Publ. Math., 51, 137-173, (1980).

[14] R. Krikorian, Global density of reducible quasi-periodic cocycles on $\mathbb{T}^{1} \times S U(2)$,Annals of Mathematics 154, 269-326, (2001).

[15] J. Moser, On invariant curves of area-preserving mappings of an annulus. Nachr. Akad. Wiss. Göttingen Math-Phys. Kl. II 1962 (1962) 1-20.

[16] L. Polterovich. The geometry of the group of symplectic diffeomorphisms. Lectures in Mathematics ETH Zürich. Birkhäuser Verlag, Basel, 2001. xii+132 pp.

[17] H. Rüssmann, Kleine Nenner I: Über invariante Kurven differenzierbarer Abbildungen eines Kreisringes, Nachr. Akad. Wiss. Gottingen, II Math.-Phys. Kl., vol. 5, pp. 67-105, (1970).

[18] S. Smale, Differentiable dynamical systems, Bull. Amer. Math. Soc. 73, 747-817, (1967).

[19] S. Sternberg, On the structure of local homeomorphisms of euclidean $n$-space II. Amer. J. Math. 80 (1958) 623-631.

[20] S. Sternberg. The structure of local homeomorphism III. Amer. J. Math. 81, 578604 (1959).

[21] D. Treschev, O. Zubelevich, Introduction to the perturbation theory of Hamiltonian systems. Springer Monographs in Mathematics. Springer-Verlag, Berlin, 2010. $\mathrm{x}+211 \mathrm{pp}$. 
[22] J.-C. Yoccoz, Conjugaison différentiable des difféomorphismes du cercle dont le nombre de rotation vérifie une condition diophantienne., Ann. Sci. École Norm. Sup. (4) 17 (1984), no. 3, 333-359.

[23] J.-C. Yoccoz, Théorème de Siegel, nombres de Bruno et polynômes quadratiques, Petits diviseurs en dimension 1. Astérisque No. 231 (1995), 3-88.

[24] J.-C. Yoccoz, Analytic linearization of circle diffeomorphisms. Dynamical systems and small divisors (Cetraro, 1998), 125-173, Lecture Notes in Math., 1784, Fond. CIME/CIME Found. Subser., Springer, Berlin, 2002.

RK: Department of Mathematics, CNRS UMr 8088, CY Cergy Paris Université (University of Cergy-Pontoise), 2, av. Adolphe Chauvin F-95302 Cergy-Pontoise, France.

Email address: raphael.krikorian@cyu.fr. 